\title{
A STOCHASTIC IMMERSED BOUNDARY METHOD FOR FLUID-STRUCTURE DYNAMICS AT MICROSCOPIC LENGTH SCALES
}

\author{
PAUL J. ATZBERGER *, PETER R. KRAMER ${ }^{\dagger}$, AND CHARLES S. PESKIN $\ddagger$
}

\begin{abstract}
In this work it is shown how the immersed boundary method of (64) for modeling flexible structures immersed in a fluid can be extended to include thermal fluctuations. A stochastic numerical method is proposed which deals with stiffness in the system of equations by handling systematically the statistical contributions of the fastest dynamics of the fluid and immersed structures over long time steps. An important feature of the numerical method is that time steps can be taken in which the degrees of freedom of the fluid are completely underresolved, partially resolved, or fully resolved while retaining a good level of accuracy. Error estimates in each of these regimes are given for the method. A number of theoretical and numerical checks are furthermore performed to assess its physical fidelity. For a conservative force, the method is found to simulate particles with the correct Boltzmann equilibrium statistics. It is shown in three dimensions that the diffusion of immersed particles simulated with the method has the correct scaling in the physical parameters. The method is also shown to reproduce a well-known hydrodynamic effect of a Brownian particle in which the velocity autocorrelation function exhibits an algebraic $\left(\tau^{-3 / 2}\right)$ decay for long times $(6 ; 16 ; 20 ; 23 ; 37 ; 38 ; 54 ; 67 ; 78)$. A few preliminary results are presented for more complex systems which demonstrate some potential application areas of the method.
\end{abstract}

Key words. Stochastic Processes, Fluid Dynamics, Brownian Dynamics, Statistical Mechanics, Immersed Boundary Method, Brownian Ratchet, Polymer Knot, Osmotic Pressure

1. Introduction. In modeling many biological systems it is important to take into account the interaction of flexible structures with a fluid. The immersed boundary method of (64) has found wide use as an efficient numerical method for simulating such systems. Some examples include the study of blood flow around heart valves (65), wave propagation in the inner ear (32), and the generation of lift in insect flight (56). With experimental advances in molecular and cellular biology has come an increasing interest in developing methods to model qualitatively and quantitatively microscopic biological processes at the cellular and subcellular level $(12 ; 27 ; 41)$. The immersed boundary method provides a promising framework for simulating such systems.

At the cellular level the fluid may consist of either the aqueous environment outside of the cell or the cytoplasm within. Some important flexible structures in the cellular context include the outer cell membrane, intracellular vesicles, cytoskeletal fibers, and molecular motor proteins. These structures play an important role in cell motility or cell division among other processes (8).

In such systems the relevant features can span a range of length scales from tens of microns or more for the outer cell membrane and cytoskeletal fibers to tens of nanometers for individual cytoskeletal monomers and motor proteins. At these length scales thermal fluctuations of the system become significant and in many cases appear crucial to achieve biological function. Some examples include force generation and progression of molecular motors along cytoskeletal fibers $(1 ;$ 41; 84), osmotic effects such as vesicle and gel swelling $(26 ; 33 ; 51 ; 61 ; 83)$, and polymerization effects

\footnotetext{
${ }^{*}$ University of California, Department of Mathematics , Santa Barbara, CA 93106; e-mail: atzberg@math.ucsb.edu; phone: 805-679-1330; Work supported by NSF Grant DMS - 9983646 and DMS-0635535.

${ }^{\dagger}$ Rensselaer Polytechnic Institute, Department of Mathematics , Troy, NY 12180; e-mail: kramep@rpi.edu; phone 518-276-6896; Work supported by NSF CAREER DMS - 0449717.

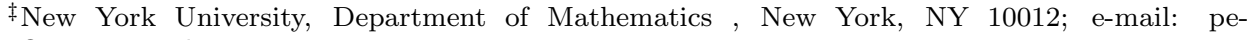
skin@cims.nyu.edu.
} 
involved in force generating processes in cell motility $(34 ; 60 ; 72 ; 81)$.

Modeling such complex cellular systems in molecular detail is infeasible with methods such as molecular dynamics as a consequence of the immense computational cost required to resolve the broad range of active length and time scales. This suggests that a coarse-grained numerical approach must be taken which does not resolve all of the detailed physics but rather attempts to make approximations that yield effective equations to capture the most relevant features of the dynamical phenomenon being studied. Here we discuss how the framework of the immersed boundary method can be extended for use in such modeling by including thermal fluctuations to capture dynamical phenomena at the cellular length scale.

The theory of nonequilibrium statistical mechanics indicates that the influence of thermal fluctuations on a mechanical system can typically be represented through the addition of thermal forcing terms which decorrelate rapidly in time. The forcing can then be represented by appropriate "white noise" processes. This generally involves a nontrivial structure of correlations between the state variables in such a way that there is an energy balance between the thermal forcing and dissipation of the system so that a corresponding fluctuation-dissipation theorem for the system is satisfied (47; 70).

Several computational fluid dynamical schemes have been extended toward the microscale through such an inclusion of thermal forces. The most widely used approach is known as Stokesian or Brownian dynamics $(15 ; 22 ; 75 ;$;7). In this approach the structures are modeled as collections of rigid "elementary particles" which interact through force laws derived by approximating the fluid dynamics by a quasi-steady Stokes flow. This latter approximation is strictly appropriate only when, among other assumptions, the fluid density is much less than the density of the structures (19), a condition better met in engineering applications (such as suspensions $(14 ; 15 ; 77)$ ) than in physiological settings (13). The result of the underlying approximations in the Stokesian/Brownian dynamics method is a rather strongly coupled system of stochastic equations for the motion of the elementary particles. For a well-designed computation the cost of a simulation can be rendered roughly proportional to $N \log N$, where $N$ is the number of elementary particles (77). In the presence of fast time scales arising from thermal fluctuations and possibly chemically activated processes, the impact of the quasi-steady Stokes approximation and the representation of the elementary particles as rigid (rather than flexible) on the accuracy of the simulation is not yet clear $(\underline{73})$.

Another approach for modeling fluids with immersed structures is Dissipative Particle Dynamics $(9 ;$ 18; 24; 25; 35; 39; $53 ;$ 58; 63). The method is built phenomenologically in terms of "fluid particles" which represent a parcel of fluid along with its collection of immersed structures. When thermal forces are included in the method the fluid particles are simulated with a stochastic system of equations modeling their (soft) interactions. This method however does not readily extend to the microscopic domain since the immersed structures within a parcel are not resolved in detail. Dissipative Particle Dynamics may however be appropriate for somewhat larger scale simulations in which one is interested in the effects of a numerous collection of immersed polymers or other structures on the dynamics of a fluid flow.

A different class of approaches which emphasize the role of the fluid dynamics while making other simplifications has also been proposed. These include finiteelement ( $\underline{76}$ ) and lattice-Boltzmann (48) methods, in which the computational fluid dynamics are extended to include thermal forces in the fluid equations following the 
framework of (49). The immersed boundary method which we shall discuss belongs to this broad class of methods (45). A theoretical approach with certain similarities to the immersed boundary method with thermal fluctuations has been proposed in (62), but differs in how thermal forces are treated for the immersed structures. A virtue of the immersed boundary method when compared to other methods is the straightforward physical manner in which it approximates the interaction of the fluid with the flexible structures.

A key feature of the immersed boundary method, distinguishing it from Stokesian/Brownian dynamics, is that the dynamics of the fluid are represented in the immersed boundary equations so that subtle inertial effects of the fluid can be incorporated into the thermally fluctuating dynamics. For example, as demonstrated in Subsection 5.2. the method captures the slow decay $\left(\tau^{-3 / 2}\right)$ in the tail of the autocorrelation function of the velocity of an immersed particle. Another advantage of tracking the fluid dynamics is the natural way in which the immersed boundary method can respect the topology of flexible structures so that, for example, polymers do not cross themselves or each other. This feature gives the immersed boundary method the potential for efficient simulation of polymer links and knots.

A basic description of how thermal fluctuations can be incorporated within the immersed boundary method was presented in (45), and theoretical analysis of the physical behavior of the method through an asymptotic stochastic mode reduction calculation was developed in (46). The immersed boundary method was found in these theoretical works to produce generally the correct physical behavior for the thermal fluctuations of immersed structures.

Here we present a derivation for the thermal fluctuations of the immersed boundary method in the context of the time dependent Stokes equations. We then present a new numerical method developed from a novel time discretization of the stochastic equations. In addition, further theoretical analysis of the framework is performed to investigate the physical behavior of the method and comparisons are made between theory and numerical simulations.

For stochastic differential equations most traditional finite difference methods, such as Runge-Kutta, achieve a lower order of accuracy than for deterministic ordinary differential equations as a consequence of the nondifferentiability of Brownian motion and its order $t^{1 / 2}$ scaling in time (44). When considering the full system of equations of the immersed boundary method, these issues are further compounded by a wide range of length and time scales that arise in many problems.

For small length scale systems in which the Reynolds number is small and the fluid flow is Stokesian to a good approximation, the time scales associated with the fine-scale fluid modes can be considerably faster than the time scales of the largescale fluid modes and the immersed structures. In many problems it is the dynamics of the immersed structures and the large-scale features of the fluid flow that are of interest. The fine-scale features of the fluid are incorporated in simulations primarily to determine their effects on the larger scales but are often not of direct interest in and of themselves.

Since the full system is rather stiff as a result of the fast time scales of the finescale fluid modes, we would like to be able to take time steps which underresolve those scales of the fluid which are not of primary interest in the simulation. However, we must take care in how this is done, because otherwise the effects of the underresolved fluid modes on the more interesting degrees of freedom can be misrepresented. For example, the simple use of a method such as Euler-Marayama (44) leads to poor 
accuracy for the stochastic dynamics when long time steps are used. In this case, the particle trajectories are found to be overly diffuse in the sense that the wrong scaling is obtained for the mean squared distance traveled.

After a presentation of the general framework of the immersed boundary method without spatiotemporal discretization in Section 2 a stochastic analysis is developed in Section 3 to design a numerical method which maintains accuracy over time steps which can be longer than the time scales of some or all of the fluid modes. The systematically derived numerical scheme presented here improves upon in several ways a correction-factor approach taken in (45) to achieve long time steps. The numerical method is constructed from a new time discretization of the immersed boundary equations, in which the equations are integrated analytically using standard techniques from stochastic calculus under well controlled approximations. The numerical method allows for the statistical contributions of the fast stochastic dynamics of the fluid, which are not explicitly resolved over long time steps, to be accounted for in the dynamics of the immersed structures. Moreover, the correlations in the statistics between the fluctuations of the degrees of freedom of the system are handled systematically, allowing for consistent realizations of the velocity field of the fluid and immersed structures to be simulated.

In Section 4 error estimates are given which indicate that the method attains a good level of accuracy (in a strong statistical sense (44)) whether the fluid modes are completely underresolved, partially resolved, or fully resolved. Only the degrees of freedom of the immersed structures constrain the time step. The numerical method handles a broad range of time steps in a unified manner, so that depending on the application, the fast dynamics of the fluid can either be explicitly resolved or underresolved, with their effects correctly represented on the structural degrees of freedom.

In Section 5, an expression for the diffusion coefficient of immersed particles is derived. The predictions are compared with the results of numerical simulations showing good agreement with the theory for different particle sizes and both short and long time steps. It is further shown that for intermediate time steps, the method captures a well-known hydrodynamic effect of a Brownian particle, in which the decay of the autocorrelation function of the velocity of the particle decays algebraically $\left(\tau^{-3 / 2}\right) \quad(6 ; ; 16 ; 20 ; 23 ; 37 ; 38 ; 54 ; 67 ; 78)$. Also in Section 5 numerical simulations are performed which confirm that the method produces the correct osmotic pressure and equilibrium statistical distribution for the position of particles within an external potential at finite temperature. To demonstrate more complex applications of the method, simulations are then presented which investigate behavior of the osmotic pressure associated with confinement of molecular dimers and polymer knots in a microscopic chamber, as well as a basic model of a molecular motor protein immersed in a fluid subject to a hydrodynamic load force.

The physical consistencies we demonstrate in the method through our analysis and numerical experiments indicate that the stochastic immersed boundary method is a viable means to model on a coarse scale the influence of thermal fluctuations on the interaction of fluids and flexible structures. This suggests that the method holds promise as an effective approach in modeling complex biological phenomena which operate at the cellular and subcellular level.

2. Fluid-Particle Equations. For the physical systems with which we shall be concerned, the relevant length scales will typically be on the order of tens of microns or smaller. The amplitude of the velocity fluctuations on these scales are sufficiently small, relative to the viscosity and length scale, that the Reynolds number is very 
small. This will allow us to neglect the nonlinear advection term in the Navier-Stokes equation for the fluid dynamics. However, we will not drop the time derivative term (as is often done in low Reynolds number limits $(50)$ ) because the dynamical time scales arising from Brownian motion and possibly certain vibrational modes of the immersed structures are in general too fast to allow this. That is, the dynamics can generally exhibit time scales which are much shorter than the advection time scale (length scale divided by velocity scale), so we can drop the nonlinear advection term but not the time derivative of the velocity. This leads us to the time-dependent Stokes equations for an incompressible fluid, which read

$$
\begin{aligned}
\rho \frac{\partial \mathbf{u}(\mathbf{x}, t)}{\partial t} & =\mu \Delta \mathbf{u}(\mathbf{x}, t)-\nabla p+\mathbf{f}_{\text {total }}(\mathbf{x}, t) \\
\nabla \cdot \mathbf{u} & =0
\end{aligned}
$$

where $p$ is the pressure arising from the incompressibility constraint, $\rho$ is the fluid density, $\mu$ is the dynamic viscosity, and $\mathbf{f}_{\text {total }}$ is the total force density acting on the fluid.

The force density acting on the fluid arises from two sources. The first source is the forces applied to the fluid by the immersed structures and particles. This component of the force density, denoted $\mathbf{f}_{\mathrm{prt}}$, generally arises from the elastic deformations of immersed structures, but they may also be applied externally and transmitted by the immersed structures to the fluid. The second contribution to the force density is from the thermal fluctuations of the system and is denoted by $\mathbf{f}_{\mathrm{thm}}$. Each of these force densities will be discussed in greater detail below. Together, they comprise the total force density acting on the fluid

$$
\mathbf{f}_{\text {total }}(\mathbf{x}, t)=\mathbf{f}_{\text {prt }}(\mathbf{x}, t)+\mathbf{f}_{\mathrm{thm}}(\mathbf{x}, t) .
$$

The immersed boundary model for fluid-structure and fluid-particle coupling treats the flexible structures and particles to first approximation as part of the fluid, representing their structural properties (such as elasticity) through the force density term $\mathbf{f}_{\text {prt }}$ (64). All structures, such as membranes, polymers, and particles, are modeled as a collection of $M$ discrete "elementary particles," with locations denoted by $\left\{\mathbf{X}^{[j]}(t)\right\}_{j=1}^{M}$, which interact with force laws appropriate to their structural properties. For simplicity, we consider the case in which all forces can be described in terms of a conservative potential $V(\{\mathbf{X}\})$ depending on the positions of the collection of elementary particles. More general force relations, including active forces, can be included (64). For notational convenience in the exposition, the range of indices for the collection of elementary particles will often be omitted. We will often refer to the forces exerted by the immersed structures as "particle forces," since the structures are represented in the numerical method as a collections of interacting particles.

In the immersed boundary method, elementary particles of size $a$ are represented by a function $\delta_{a}(\mathbf{x})$ which may be thought of as a Dirac delta function smoothed over a length scale $a$ in such a manner that the smoothed delta function has good numerical properties (See Appendix @ and (64)).

This smoothed delta function is used both in converting the force associated with an elementary particle to a localized force density acting on the fluid:

$$
\mathbf{f}_{\mathrm{prt}}(\mathbf{x}, t)=\sum_{j^{\prime}=1}^{M}\left(-\nabla_{\mathbf{X}^{\left[j^{\prime}\right]}} V\right)(\{\mathbf{X}(t)\}) \delta_{a}\left(\mathbf{x}-\mathbf{X}^{\left[j^{\prime}\right]}(t)\right)
$$


and in computing the velocity of the elementary particle by an interpolation of the fluid velocity in its vicinity:

$$
\frac{d \mathbf{X}^{[j]}(t)}{d t}=\int_{\Lambda} \delta_{a}\left(\mathbf{x}-\mathbf{X}^{[j]}(t)\right) \mathbf{u}(\mathbf{x}, t) d \mathbf{x} .
$$

The integration is over the entire domain $\Lambda$ of the fluid. In the immersed boundary formulation, this includes the space occupied by the immersed particles and structures, which are thought of as parts of the fluid in which additional forces happen to be applied. In particular, the domain $\Lambda$ is independent of time, despite the motion of the immersed material.

We also note that in the present context the parameter $a$ is a physical parameter of the model, since it is supposed to represent a physical dimension of an elementary particle. In particular, $a$ is not a numerical parameter which is supposed to vanish along with the meshwidth for the fluid computations as it is refined. In this respect, the use of smoothed delta functions described here is different from the standard use of such functions in immersed boundary computations. The idea that smoothed delta functions could be used to model the physical dimensions of immersed objects was previously proposed in (55) under the name "force cloud method".

3. Numerical Method. We shall now discuss a numerical discretization and specification of the thermal force density for the equations 2.1 2.5 defining the immersed boundary method. A summary of the numerical method in algorithmic form is given in Subsection 3.1, followed by a heuristic discussion in Subsection 3.2 and a mathematical derivation in Subsection 3.3 .

3.1. Summary of the Numerical Method. The numerical method is based upon a finite difference discretization of the differential equations 2.1 2.5 describing the coupled dynamics of the fluid and the immersed structures. The fluid variables (velocity field $\mathbf{u}$ and pressure field $p$ ) are represented on a periodic grid with length $L$ along each direction, $N$ grid points along each direction, and grid spacing $\Delta x=L / N$. The values of these fields on the lattice will be denoted through subscripted variables such as $\mathbf{u}_{\mathbf{m}}$ and $p_{\mathbf{m}}$, where the subscript $\mathbf{m}=\left(m_{1}, m_{2}, m_{3}\right)$ is a vector with integer components indicating the grid point in question (relative to some arbitrarily specified origin). The position of the grid point with index $\mathbf{m}$ is denoted by $\mathbf{x}_{\mathbf{m}}$.

The Discrete Fourier Transform (DFT) of the fluid variables plays an important role in the numerical simulation scheme, and is related to the physical space values on the grid through the formulas:

$$
\begin{aligned}
\hat{\mathbf{u}}_{\mathbf{k}} & =\frac{1}{N^{3}} \sum_{\mathbf{m}} \mathbf{u}_{\mathbf{m}} \exp (-i 2 \pi \mathbf{k} \cdot \mathbf{m} / N) \\
\mathbf{u}_{\mathbf{m}} & =\sum_{\mathbf{k}} \hat{\mathbf{u}}_{\mathbf{k}} \exp (i 2 \pi \mathbf{k} \cdot \mathbf{m} / N)
\end{aligned}
$$

where each of the sums in the above equations runs over the $N^{3}$ lattice points defined by $0 \leq \mathbf{k}^{(\ell)} \leq N-1$, and $0 \leq \mathbf{m}^{(\ell)} \leq N-1$, where the parenthesized superscripts $\ell=1,2,3$ denote the Cartesian components of the indicated vector. In fact any translate of these blocks of lattice points could be used equivalently in the sums, due to the underlying periodicity.

Time is discretized into time steps $\Delta t$, and the values of the system variables at the $n^{\text {th }}$ time step, corresponding to the time $t_{n}=n \Delta t$, are denoted with a superscript 
integer $n$. The procedure by which these variables are updated from one time step to the next is now described:

1. The structural forces exerted by the immersed structures are computed and the lattice values of the particle force density field which is applied to the fluid is obtained from

$$
\mathbf{f}_{\mathbf{m}}^{n}=\sum_{j^{\prime}=1}^{N}-\left(\nabla_{\mathbf{X}^{\left[j^{\prime}\right]}} V\right)\left(\left\{\mathbf{X}^{n}\right\}\right) \delta_{a}\left(\mathbf{x}_{\mathbf{m}}-\mathbf{X}^{n,\left[j^{\prime}\right]}\right) .
$$

Here and afterwards, we drop the subscript "prt" from the particle force density. The Fourier coefficients $\hat{\mathbf{f}}_{\mathbf{k}}^{n}$ of this particle force density field are computed using a Fast Fourier Transform (FFT).

2. The Fourier coefficients of the velocity field of the fluid are updated by the stochastic recurrence

$$
\hat{\mathbf{u}}_{\mathbf{k}}^{n+1}=e^{-\alpha_{\mathbf{k}} \Delta t} \hat{\mathbf{u}}_{\mathbf{k}}^{n}+\frac{1}{\rho \alpha_{\mathbf{k}}}\left(1-e^{-\alpha_{\mathbf{k}} \Delta t}\right) \wp_{\mathbf{k}}^{\perp} \hat{\mathbf{f}}_{\mathbf{k}}^{n}+\wp_{\mathbf{k}}^{\perp} \hat{\Xi}_{\mathbf{k}}^{n},
$$

where $\wp_{\mathbf{k}}^{\perp}$ denotes the projection orthogonal to $\hat{\mathbf{g}}_{\mathbf{k}}$ defined by

$$
\hat{\mathbf{g}}_{k}^{(j)}=\sin \left(2 \pi \mathbf{k}^{(j)} / N\right) / \Delta x
$$

which is used to enforce the incompressibility constraint 2.2. The factor $\hat{\Xi}_{\mathbf{k}}^{n}=\sigma_{\mathbf{k}} \tilde{\eta}_{\mathbf{k}}$ accounts for the thermal fluctuations over the time step, where $\tilde{\boldsymbol{\eta}}_{\mathbf{k}}$ denotes a complex vector-valued random variable independent in $\mathbf{k}$, having independent real and imaginary components, each of which are Gaussian random variables with mean zero and variance one. The variance of $\hat{\boldsymbol{\Xi}}_{\mathbf{k}}^{n}$ is determined in Subsection 3.3 .4 and is given by

$$
\sigma_{\mathbf{k}}^{2}=\frac{D_{\mathbf{k}}}{\alpha_{\mathbf{k}}}\left(1-\exp \left(-2 \alpha_{\mathbf{k}} \Delta t\right)\right),
$$

where

$$
\left.\alpha_{\mathbf{k}}=\frac{2 \mu}{\rho \Delta x^{2}} \sum_{j=1}^{3}\left(1-\cos \left(2 \pi \mathbf{k}^{(j)} / N\right)\right)\right)
$$

and

$$
D_{\mathbf{k}}= \begin{cases}\frac{k_{B} T}{\rho L^{3}} \alpha_{\mathbf{k}} & , \mathbf{k} \in \mathcal{K} \\ \frac{k_{B} T}{2 \rho L^{3}} \alpha_{\mathbf{k}} & , \mathbf{k} \notin \mathcal{K}\end{cases}
$$

with

$$
\mathcal{K}=\left\{\mathbf{k} \mid \mathbf{k}^{(j)}=0 \text { or } \mathbf{k}^{(j)}=N / 2, j=1,2,3\right\} .
$$

3. The elementary particle positions are updated by

$$
\mathbf{X}^{n+1,[j]}-\mathbf{X}^{n,[j]}=\sum_{\mathbf{m}} \delta_{a}\left(\mathbf{x}_{\mathbf{m}}-\mathbf{X}^{n,[j]}\right) \boldsymbol{\Gamma}_{\mathbf{m}}^{n} \Delta x^{3},
$$


where $\boldsymbol{\Gamma}_{\mathbf{m}}^{n}$ is the time integrated velocity field of the fluid. It is obtained by a discrete Inverse Fast Fourier Transform (IFFT) of appropriately generated random variables $\hat{\boldsymbol{\Gamma}}_{\mathbf{k}}^{n}$ in Fourier space:

$$
\boldsymbol{\Gamma}_{\mathbf{m}}^{n}=\int_{t_{n}}^{t_{n+1}} \mathbf{u}_{\mathbf{m}}(s) d s=\sum_{\mathbf{k}} \hat{\boldsymbol{\Gamma}}_{\mathbf{k}}^{n} \cdot \exp (i 2 \pi \mathbf{k} \cdot \mathbf{m} / N) .
$$

The $\hat{\boldsymbol{\Gamma}}_{\mathbf{k}}^{n}$ are computed from

$$
\hat{\boldsymbol{\Gamma}}_{\mathbf{k}}^{n}=\hat{\mathbf{H}}_{\mathbf{k}}+c_{1, \mathbf{k}} \wp_{\mathbf{k}}^{\perp} \hat{\Xi}_{\mathbf{k}}^{n}+c_{2, \mathbf{k}} \wp_{\mathbf{k}}^{\perp} \hat{\mathbf{G}}_{\mathbf{k}},
$$

where $\hat{\boldsymbol{\Xi}}_{\mathbf{k}}^{n}$ is obtained from step 2 and $\hat{\mathbf{H}}_{\mathbf{k}}$ is computed from steps 1 and 2 by

$$
\hat{\mathbf{H}}_{\mathbf{k}}=\frac{1-\exp \left(-\alpha_{\mathbf{k}} \Delta t\right)}{\alpha_{\mathbf{k}}} \hat{\mathbf{u}}_{\mathbf{k}}^{n}+\left(\frac{\Delta t}{\alpha_{\mathbf{k}}}+\left(\frac{1}{\alpha_{\mathbf{k}}}\right)^{2}\left(\exp \left(-\alpha_{\mathbf{k}} \Delta t\right)-1\right)\right) \rho^{-1} \wp_{\mathbf{k}}^{\perp} \hat{\mathbf{f}}_{\mathbf{k}}^{n} .
$$

The random variable $\hat{\mathbf{G}}_{\mathbf{k}}$ is computed from scratch for each mode $\mathbf{k}$ by generating a complex vector-valued random variable having independent real and imaginary components, each of which are Gaussian random variables with mean zero and variance one. The constants in 3.12 are given by

$$
c_{1, \mathbf{k}}=\frac{1}{\alpha_{\mathbf{k}}} \tanh \left(\frac{\alpha_{\mathbf{k}} \Delta t}{2}\right)
$$

and

$$
c_{2, \mathbf{k}}=\sqrt{\left(\frac{2 D_{\mathbf{k}}}{\alpha_{\mathbf{k}}^{3}}\right)\left(\alpha_{\mathbf{k}} \Delta t-2 \tanh \left(\frac{\alpha_{\mathbf{k}} \Delta t}{2}\right)\right)} .
$$

In this manner the time integrated velocity field is consistently generated with the correct correlations with $\left\{\mathbf{u}_{\mathbf{k}}^{n}\right\}$ and $\left\{\mathbf{u}_{\mathbf{k}}^{n+1}\right\}$ from steps 1 and 2. For more details, see Subsections 3.3.5 and 3.3.6.

The computational complexity of the method, when excluding the application specific forces acting on the immersed structures, is dominated by the FFT and IFFT, which for a three dimensional lattice requires $O\left(N^{3} \log (N)\right)$ arithmetic steps.

3.2. Heuristic Discussion of the Numerical Method. We now briefly discuss each step of the numerical scheme to give some intuition into how the method operates. A more rigorous mathematical discussion and derivation is given in Subsection 3.3 .

The first step of the numerical scheme computes the structural forces exerted by the elementary particles as a function of their configuration, and computes the discrete Fourier transform of the corresponding force density field acting on the fluid. The second step updates the fluid velocity in Fourier space by integrating over the structural forces and thermal forces experienced over a time step. The appearance of the time step $\Delta t$ in exponential factors is due to the design of the method to maintain accuracy even if the fluid dynamics are partially resolved or underresolved by the time step, as in "exponential time differencing" schemes $(30 ; 36 ; 42)$. The key time scale paired against the time step in these formulas is $1 / \alpha_{\mathbf{k}}$, which describes the 
time scale of viscous damping of the Fourier mode $\mathbf{k}$ of the fluid, as simulated by the numerical method. The first term of the stochastic recurrence equation 3.4 represents the viscously dissipated contribution of the fluid velocity from the previous time step. The second term represents the contribution from the structural forces during the time step. Since the elementary particle dynamics are assumed to be resolved by the time step, the structural force density itself appears in a simple way as effectively constant over the time step. The response of the fluid velocity to this force has an exponential dependence on the time step to account for the possible levels of resolution of the viscous damping. The third term accounts for the thermal fluctuations over the time step through a mean zero Gaussian random variable $\hat{\Xi}_{\mathbf{k}}^{n}$ with variance $\sigma_{\mathbf{k}}^{2}$ describing the magnitude of the net contribution of the thermal fluctuations to the fluid velocity over the time step, see equation 3.6. Note that for time steps $\Delta t$ long compared to the relaxation time $1 / \alpha_{\mathbf{k}}$ of the velocity Fourier mode, the variance approaches the constant value $k_{B} T /\left(\rho L^{3}\right)$ corresponding to the equilibrium equipartition value. On the other hand, for $\Delta t \ll 1 / \alpha_{\mathbf{k}}$, the variance of the thermal velocity increment is proportional to $\Delta t$, so the magnitude of the increment is proportional to $\sqrt{\Delta t}$. This latter scaling is typical for the response of physical systems to noise driven by a large number of weak inputs (i.e., molecular fluctuations) (44). In both the second and third terms, the projection $\wp_{\mathbf{k}}^{\perp}$ enforces the incompressibility of the fluid. The distinction in the definition of the factor $D_{\mathbf{k}}$ with respect to wavenumbers $\mathbf{k}$, as specified by the set $\mathcal{K}$, is a purely technical issue related to the discrete Fourier transform; see Subsection 3.3.3.

The third step of the numerical method updates the positions of the elementary particle positions composing the immersed structures. The random variable $\boldsymbol{\Gamma}_{\mathbf{m}}^{n}$ represents the fluid velocity at lattice point $\mathbf{x}_{\mathbf{m}}$ integrated over the time step, and is generated in Fourier space using a procedure which also ensures that the immersed structures move with the correct correlations with the previously computed fluid velocity values $\hat{\mathbf{u}}_{\mathbf{k}}^{n}$ and $\hat{\mathbf{u}}_{\mathbf{k}}^{n+1}$. The formulas defining $\boldsymbol{\Gamma}_{\mathbf{m}}^{n}$ arise from an exact formula for integrating the fluid velocity over a time step, under the assumption that the structural forces can be treated as constant over the time step.

3.3. Derivation of the Numerical Method. The derivation first considers a spatial discretization of equation 2.1 while leaving the system of equations continuous in time to avoid technical issues associated with the continuum formulation of the stochastic immersed boundary method with thermal forcing (46). Since the equations are meant to serve as a physical model for the dynamics of the immersed structures and fluid, a thermal forcing is derived for the semi-discretized system which is consistent with equilibrium statistical mechanics in Subsection 3.3.3. The time discretization of the numerical method for both the dynamics of the fluid and immersed structures is then discussed in Subsection 3.3.4 and Subsection 3.3.5. The method takes special care to account for correlations between the dynamics of the fluid and immersed structures, which is discussed in Subsection 3.3.6. We remark that throughout the derivation, the integration steps of the numerical method are designed to maintain accuracy even when the time step does not fully resolve the dynamics of the fluid modes.

3.3.1. Semi-discretization. The equations 2.1 and 2.2 can be discretized in space by finite difference approximations for the spatial derivatives (64)

$$
\rho \frac{d \mathbf{u}_{\mathbf{m}}^{(\ell)}}{d t}=\mu \sum_{q=1}^{3} \frac{\mathbf{u}_{\mathbf{m}-\mathbf{e}_{q}}^{(\ell)}(t)-2 \mathbf{u}_{\mathbf{m}}^{(\ell)}(t)+\mathbf{u}_{\mathbf{m}+\mathbf{e}_{q}}^{(\ell)}(t)}{\Delta x^{2}}
$$




$$
\begin{gathered}
-\frac{p_{\mathbf{m}+\mathbf{e}_{\ell}}-p_{\mathbf{m}-\mathbf{e}_{\ell}}}{2 \Delta x}+\mathbf{f}_{\text {total }}^{(\ell)}\left(\mathbf{x}_{\mathbf{m}}, t\right) \\
\sum_{\ell=1}^{3} \frac{\mathbf{u}_{\mathbf{m}+\mathbf{e}_{\ell}}^{(\ell)}(t)-\mathbf{u}_{\mathbf{m}-\mathbf{e}_{\ell}}^{(\ell)}(t)}{2 \Delta x}=0
\end{gathered}
$$

where $\mathbf{e}_{\ell}$ denotes the standard basis vector with all zero entries except for a one in the $\ell^{t h}$ position. The parenthesized superscripts denote the vector component.

The equations for the fluid-particle coupling become

$$
\begin{aligned}
\mathbf{f}_{\mathrm{prt}}\left(\mathbf{x}_{\mathbf{m}}, t\right) & =\sum_{j^{\prime}=1}^{M}-\left(\nabla_{\mathbf{X}^{\left[j^{\prime}\right]}} V\right)(\{\mathbf{X}(t)\}) \delta_{a}\left(\mathbf{x}_{\mathbf{m}}-\mathbf{X}^{\left[j^{\prime}\right]}(t)\right) \\
\frac{d \mathbf{X}^{[j]}(t)}{d t} & =\mathbf{U}\left(\mathbf{X}^{[j]}(t), t\right) \\
\mathbf{U}(\mathbf{x}, t) & =\sum_{\mathbf{m}} \delta_{a}\left(\mathbf{x}_{\mathbf{m}}-\mathbf{x}\right) \mathbf{u}\left(\mathbf{x}_{\mathbf{m}}, t\right) \Delta x^{3}
\end{aligned}
$$

Since we do not take the limit $a \rightarrow 0$ as the meshwidth is refined (see above), $\mathbf{U}\left(\mathbf{X}^{[j]}(t), t\right)$ does not become the same as $\mathbf{u}\left(\mathbf{X}^{[j]}(t), t\right)$, even in the limit $\Delta x \rightarrow 0$. That is, we are not simply evaluating the fluid velocity at $\mathbf{X}^{[j]}(t)$, but instead averaging it over a region of width determined by the parameter $a$. This averaging procedure ensures that the particle velocity remains finite and well-defined in the continuum limit, in which the pointwise values of the thermally fluctuating fluid velocity diverge. Indeed, according to general statistical mechanical principles for continuum fields (47; 49; 70) (and not from any feature particular to the numerical method), the thermally fluctuating fluid velocity field manifests increasingly wilder fluctuations on smaller scales, and in the theoretical continuum limit approaches a sort of white noise structure. This is not inherently problematic for physical interpretation, which only requires that meaningful values be obtained from averages over finite volumes, such as the size of a probe or an immersed structure. Such averages are indeed finite, as can be understood intuitively through central limit theorem considerations by viewing them as averages of a large number of independent mean zero random variables due to the rapid spatial decorrelation of the noisy continuum velocity field. From a more mathematical standpoint, an application of the convolution theorem to the definition of $\mathbf{U}$ in (3.18) yields that $\mathbf{U}$ can be represented as a nicely convergent Fourier series due to the decay of the Fourier coefficients of the smooth function $\delta_{a}$.

To obtain other desirable behaviors, we also remark that it is important that we use the same weight function $\delta_{a}$ in averaging the fluid velocity as we do in applying force to the fluid, since this ensures that energy is properly conserved in the fluidparticle interaction. With appropriate care in the construction of $\delta_{a}$, one can further ensure that momentum and angular momentum are conserved as well; see (64).

3.3.2. Fluid Equations in Fourier Space. The Stokes equation is given in Fourier space by

$$
\begin{aligned}
\frac{d \hat{\mathbf{u}}_{\mathbf{k}}}{d t} & =-\alpha_{\mathbf{k}} \hat{\mathbf{u}}_{\mathbf{k}}-\mathrm{i} \rho^{-1} \hat{p}_{\mathbf{k}} \hat{\mathbf{g}}_{\mathbf{k}}+\rho^{-1} \hat{\mathbf{f}}_{\text {total }, \mathbf{k}} \\
\hat{\mathbf{g}}_{\mathbf{k}} \cdot \hat{\mathbf{u}}_{\mathbf{k}} & =0
\end{aligned}
$$


where

$$
\begin{gathered}
\left.\alpha_{\mathbf{k}}=\frac{2 \mu}{\rho \Delta x^{2}} \sum_{j=1}^{3}\left(1-\cos \left(2 \pi \mathbf{k}^{(j)} / N\right)\right)\right) \\
\hat{\mathbf{g}}_{k}^{(j)}=\sin \left(2 \pi \mathbf{k}^{(j)} / N\right) / \Delta x .
\end{gathered}
$$

Since the velocity field of the fluid is real-valued, a further condition that must be satisfied by solutions of the equations $3.21-3.22$ is

$$
\overline{\hat{\mathbf{u}}_{\mathrm{N}-\mathbf{k}}}=\hat{\mathbf{u}}_{\mathrm{k}},
$$

where $\mathbf{N}$ is shorthand for $(N, N, N)^{T}$ and the overbar denotes complex conjugation. Provided the force is real-valued, it can be shown that if this constraint holds for the initial conditions it will be satisfied for all time.

The Fourier coefficients $\hat{p}_{\mathbf{k}}(t)$ of the pressure need to be chosen in order to ensure that the incompressibility constraint is satisfied. They can be determined by projecting both sides of equation 3.21 onto $\hat{\mathrm{g}}_{\mathrm{k}}$. By the incompressibility constraint 3.22 , both of the terms involving $\hat{\mathbf{u}}_{\mathbf{k}}$ and its time derivative are zero under the projection. This gives at each time

$$
\hat{p}_{\mathbf{k}}(t)=\frac{\mathrm{i} \hat{\mathbf{g}}_{\mathbf{k}} \cdot \hat{\mathbf{f}}_{\mathrm{total}, \mathbf{k}}(t)}{\left|\hat{\mathbf{g}}_{\mathbf{k}}\right|^{2}} .
$$

For those values of $\mathbf{k}$ that make $\hat{\mathbf{g}}_{\mathbf{k}}=0$, the incompressibility constraint is trivial, and by convention we shall take $\hat{p}_{\mathbf{k}}(t)=0$ for such $\mathbf{k}$.

For future reference, let the projection in the direction $\hat{\mathbf{g}}_{\mathbf{k}}$ be denoted by

$$
\wp_{\mathbf{k}}^{\|}=\frac{\hat{\mathrm{g}}_{\mathbf{k}} \hat{\mathrm{g}}_{\mathbf{k}}^{T}}{\left|\hat{\mathrm{g}}_{\mathbf{k}}\right|^{2}}
$$

and the projection orthogonal to $\hat{\mathbf{g}}_{\mathbf{k}}$ be denoted by

$$
\wp \frac{\perp}{\mathbf{k}}=\left(\mathcal{I}-\frac{\hat{\mathbf{g}}_{\mathbf{k}} \hat{\mathbf{g}}_{\mathbf{k}}^{T}}{\left|\hat{\mathbf{g}}_{\mathbf{k}}\right|^{2}} \cdot\right)
$$

For those modes for which $\hat{\mathbf{g}}_{\mathbf{k}}=\mathbf{0}$, the corresponding projections will be understood to be defined $\wp_{\mathbf{k}}^{\|}=\mathbf{0}$ and $\wp_{\mathbf{k}}^{\perp}=\mathcal{I}$. The set of indices on which $\hat{\mathrm{g}}_{\mathbf{k}}=\mathbf{0}$ is given by

$$
\mathcal{K}=\left\{\mathbf{k} \mid \mathbf{k}^{(j)}=0 \text { or } \mathbf{k}^{(j)}=N / 2, j=1,2,3\right\} .
$$

For a function $\mathbf{w}_{\mathbf{m}}$ defined over the discrete lattice sites indexed by $\mathbf{m}$, the corresponding projection operations in physical space are given by

$$
\left(\wp^{\|} \mathbf{w}\right)_{\mathbf{m}}=\sum_{\mathbf{k}} \wp_{\mathbf{k}}^{\|} \hat{\mathbf{w}}_{\mathbf{k}} \exp (i 2 \pi \mathbf{k} \cdot \mathbf{m} / N)
$$

and

$$
\left(\wp^{\perp} \mathbf{w}\right)_{\mathbf{m}}=\sum_{\mathbf{k}} \wp_{\mathbf{k}}^{\perp} \hat{\mathbf{w}}_{\mathbf{k}} \exp (i 2 \pi \mathbf{k} \cdot \mathbf{m} / N) .
$$


3.3.3. Thermal Forcing. Following standard practice in nonequilibrium statistical mechanics $(47 ;$ 70), the thermal fluctuations of the system are modeled as Gaussian white noise. Formally, the Fourier coefficients of the thermal forcing can be written as

$$
\hat{\mathbf{f}}_{\mathrm{thm}, \mathbf{k}}=\rho \sqrt{2 D_{\mathbf{k}}} \frac{d \tilde{\mathbf{B}}_{\mathbf{k}}(t)}{d t} .
$$

The factor $D_{\mathbf{k}}$ (which is to be specified) describes the strength of the thermal forcing of the $\mathbf{k}^{t h}$ mode and $\tilde{\mathbf{B}}_{\mathbf{k}}(t)$ denotes a complex-valued Brownian motion with the real and imaginary parts of each component consisting of an independent standard Brownian motion (44). The dependence on $\mathbf{k}$ will be discussed below.

Standard Brownian motion $B(t)$ for our purposes will refer to the continuous stochastic process which is defined by the following properties:

(i) $B(0)=0$,

(ii) $E\left(B\left(t_{2}\right)-B\left(t_{1}\right)\right)=0$,

(iii) $E\left(\left|B\left(t_{2}\right)-B\left(t_{1}\right)\right|^{2}\right)=\left|t_{2}-t_{1}\right|$,

(iv) The increments $B\left(t_{2}\right)-B\left(t_{1}\right)$ and $B\left(t_{4}\right)-B\left(t_{3}\right)$ are independent Gaussian random variables whenever $t_{1}<t_{2} \leq t_{3}<t_{4}$,

where $E(\cdot)$ denotes the expected value. Standard Brownian motion in $d$ dimensions is defined as a stochastic process where each vector component is an independent onedimensional Brownian motion. For a further discussion of the properties of Brownian motion and related technical issues, see (59) or (31).

The discretized Stokes equation 3.21 with only thermal forcing (no immersed structural forces) can be expressed in stochastic differential notation as

$$
\begin{aligned}
d \hat{\mathbf{u}}_{\mathbf{k}} & =\left[-\alpha_{\mathbf{k}} \hat{\mathbf{u}}_{\mathbf{k}}-i \rho^{-1} \hat{p}_{\mathbf{k}} \hat{\mathbf{g}}_{\mathbf{k}}\right] d t+\sqrt{2 D_{\mathbf{k}}} d \tilde{\mathbf{B}}_{\mathbf{k}}(t) \\
\hat{\mathbf{g}}_{\mathbf{k}} \cdot \hat{\mathbf{u}}_{\mathbf{k}} & =0 \\
\hat{\mathbf{u}}_{\mathbf{N}-\mathbf{k}} & =\hat{\mathbf{u}}_{\mathbf{k}},
\end{aligned}
$$

where $d \tilde{\mathbf{B}}_{\mathbf{k}}(t)$ denotes increments of the complex-valued Brownian motion associated with the $\mathbf{k}^{\text {th }}$ mode. To ensure that the thermal forcing be real-valued, the Brownian increments are correlated in $\mathbf{k}$ by the constraint

$$
\overline{d \tilde{\mathbf{B}}_{\mathbf{N}-\mathbf{k}}}=d \tilde{\mathbf{B}}_{\mathbf{k}} .
$$

As discussed in Section 3.3.2 the pressure can be expressed in terms of the force acting on the fluid using 3.26. By formal substitution into 3.33 the incompressibility constraint can be incorporated through an appropriate projection operation which allows for the two equations 3.33 and 3.34 to be expressed as the single equation

$$
d \hat{\mathbf{u}}_{\mathbf{k}}+\alpha_{\mathbf{k}} \hat{\mathbf{u}}_{\mathbf{k}} d t=\sqrt{2 D_{\mathbf{k}}} \wp_{\mathbf{k}}^{\perp} d \tilde{\mathbf{B}}_{\mathbf{k}}(t) .
$$

Since the incompressibility constraint is equivalent to $\wp_{\mathbf{k}}^{\perp} \hat{\mathbf{u}}_{\mathbf{k}}=\hat{\mathbf{u}}_{\mathbf{k}}$, the constraint will be satisfied for all time provided it holds at the initial time. Consequently, when $\hat{\mathbf{g}}_{\mathbf{k}} \neq \mathbf{0}(\mathbf{k} \notin \mathcal{K}$ as defined in 3.29), the real and imaginary part of the stochastic process $\hat{\mathbf{u}}_{\mathbf{k}}(t)$ remain in the plane orthogonal to $\hat{\mathbf{g}}_{\mathbf{k}}$ for all time. When $\hat{\mathbf{g}}_{\mathbf{k}}=\mathbf{0}(\mathbf{k} \in \mathcal{K})$, no constraint is imposed on the real part, but the real-valuedness condition 3.25 requires that the imaginary component vanish. 
Equation 3.37 can be solved by the method of integrating factors to obtain

$$
\hat{\mathbf{u}}_{\mathbf{k}}(t)=\sqrt{2 D_{\mathbf{k}}} \wp_{\mathbf{k}}^{\perp} \int_{-\infty}^{t} e^{-\alpha_{\mathbf{k}}(t-s)} d \tilde{\mathbf{B}}_{\mathbf{k}}(s) .
$$

Since $\hat{\mathbf{u}}_{\mathbf{k}}(t)$ is the projection of an Ito integral with deterministic integrand, it is at each time $t$ a Gaussian random variable with mean zero.

The variance of $\hat{\mathbf{u}}_{\mathbf{k}}(t)$ can be computed from 3.38 by

$$
\begin{aligned}
E\left(\left|\hat{\mathbf{u}}_{\mathbf{k}}\right|^{2}\right) & =\operatorname{Tr}\left(E\left(\hat{\mathbf{u}}_{\mathbf{k}}{\overline{\hat{\mathbf{u}}_{\mathbf{k}}}}^{T}\right)\right) \\
& =2 D_{\mathbf{k}} \operatorname{Tr}\left(\wp_{\mathbf{k}}^{\perp} \int_{-\infty}^{t} \int_{-\infty}^{t} e^{-\alpha_{\mathbf{k}}\left(2 t-s-s^{\prime}\right)} E\left(d \tilde{\mathbf{B}}_{\mathbf{k}}(s) \overline{d \tilde{\mathbf{B}}_{\mathbf{k}}^{T}\left(s^{\prime}\right)}\right)\right)
\end{aligned}
$$

To proceed further, we must distinguish between the cases $\mathbf{k} \in \mathcal{K}$ and $\mathbf{k} \notin \mathcal{K}$, where $\mathcal{K}$ is defined in 3.29

For $\mathbf{k} \in \mathcal{K}$, we have $\wp_{\mathbf{k}}^{\perp}=\mathcal{I}$ and from constraint 3.36 that the Brownian motion $\tilde{\mathbf{B}}_{\mathbf{k}}(s)$ is real valued. Therefore,

$$
E\left(d \tilde{\mathbf{B}}_{\mathbf{k}}(s) \overline{d \tilde{\mathbf{B}}_{\mathbf{k}}^{T}\left(s^{\prime}\right)}\right)=\mathcal{I} \delta\left(s-s^{\prime}\right) d s d s^{\prime},
$$

and it follows that

$$
E\left(\left|\hat{\mathbf{u}}_{\mathbf{k}}\right|^{2}\right)=\frac{3 D_{\mathbf{k}}}{\alpha_{\mathbf{k}}}
$$

when $\mathbf{k} \in \mathcal{K}$.

For $\mathbf{k} \notin \mathcal{K}$, the Brownian motion is complex valued and $\wp_{\mathbf{k}}^{\perp}$ is a projection onto the two-dimensional subspace orthogonal to $\hat{\mathrm{g}}_{\mathbf{k}}$. Therefore,

$$
E\left(d \tilde{\mathbf{B}}_{\mathbf{k}}(s) \overline{d \tilde{\mathbf{B}}_{\mathbf{k}}^{T}\left(s^{\prime}\right)}\right)=2 \wp_{\mathbf{k}}^{\perp} \delta\left(s-s^{\prime}\right) d s d s^{\prime}
$$

and

$$
\operatorname{Tr}\left(\wp_{\mathbf{k}}^{\perp}\right)=2,
$$

from which it follows that

$$
E\left(\left|\hat{\mathbf{u}}_{\mathbf{k}}\right|^{2}\right)=\frac{4 D_{\mathbf{k}}}{\alpha_{\mathbf{k}}}
$$

for $\mathbf{k} \notin \mathcal{K}$. We remark that the formal calculations above can be justified rigorously by applying Ito's Isometry directly to equation 3.39, see reference (59).

To determine $D_{\mathbf{k}}$, we shall now compare these results with those that are obtained if we impose the condition that the immersed boundary method exhibit fluctuations governed by the Boltzmann distribution, as required by classical statistical mechanics. By Parseval's Lemma, the total kinetic energy can be expressed in terms of the Fourier modes of the fluid by

$$
\begin{aligned}
\mathcal{E}\left[\left\{\mathbf{u}_{\mathbf{k}}\right\}\right] & =\frac{\rho}{2} \sum_{\mathbf{m}}\left|\mathbf{u}_{\mathbf{m}}\right|^{2} \Delta x^{3} \\
& =\frac{\rho}{2} \sum_{\mathbf{k}}\left|\hat{\mathbf{u}}_{\mathbf{k}}\right|^{2} L^{3} .
\end{aligned}
$$


The density of the Boltzmann distribution is then given by

$$
\tilde{\Psi}\left(\left\{\hat{\mathbf{u}}_{\mathbf{k}}\right\}\right)=\frac{1}{\tilde{Z}} \exp \left(-\frac{\rho L^{3} \sum_{\mathbf{k}}\left|\hat{\mathbf{u}}_{\mathbf{k}}\right|^{2}}{2 k_{B} T}\right),
$$

where $\tilde{Z}$ is the partition function obtained by integrating $\hat{\mathbf{u}}_{\mathbf{k}}$ over the constrained subspace

$$
\Omega=\left\{\left\{\hat{\mathbf{u}}_{\mathbf{k}}\right\} \mid \hat{\mathbf{g}}_{\mathbf{k}} \cdot \hat{\mathbf{u}}_{\mathbf{k}}=0, \overline{\hat{\mathbf{u}}_{\mathbf{N}-\mathbf{k}}}=\hat{\mathbf{u}}_{\mathbf{k}}\right\} .
$$

Each degree of freedom of the fluid contributes a quadratic term to the energy of the system, giving a Boltzmann distribution which is Gaussian. Therefore, the equipartition theorem holds and each independent degree of freedom contributes on average $\frac{1}{2} k_{B} T$ to the kinetic energy.

For a particular wavenumber $\mathbf{k} \in \mathcal{K}$, the mean contribution to the energy is

$$
\frac{\rho L^{3}}{2} \frac{3 D_{\mathbf{k}}}{\alpha_{\mathbf{k}}}
$$

where the expression 3.41 for $E\left(\left|\hat{\mathbf{u}}_{\mathbf{k}}\right|^{2}\right)$ has been used. For such wavenumbers there are 3 independent degrees of freedom corresponding to the 3 real components of $\hat{\mathbf{u}}_{\mathbf{k}}$. By the equipartition theorem this requires

$$
\frac{\rho L^{3}}{2} \frac{3 D_{\mathbf{k}}}{\alpha_{\mathbf{k}}}=\frac{3}{2} k_{B} T
$$

which gives

$$
D_{\mathbf{k}}=\alpha_{\mathbf{k}} \frac{k_{B} T}{\rho L^{3}}
$$

when $\mathbf{k} \in \mathcal{K}$.

For $\mathbf{k} \notin \mathcal{K}$, we must consider the pair $(\mathbf{k}, \mathbf{N}-\mathbf{k})$ together, since $\hat{\mathbf{u}}_{\mathbf{k}}=\overline{\hat{\mathbf{u}}_{\mathbf{N}-\mathbf{k}}}$. The contribution to the mean energy of these two wavenumbers is

$$
2 \frac{\rho L^{3}}{2} \frac{4 D_{\mathbf{k}}}{\alpha_{\mathbf{k}}}
$$

where the expression 3.44 for $E\left(\left|\hat{\mathbf{u}}_{\mathbf{k}}\right|^{2}\right)$ and $E\left(\left|\hat{\mathbf{u}}_{\mathbf{N}-\mathbf{k}}\right|^{2}\right)$ has been used. The number of independent degrees of freedom corresponding to the pair of wavenumbers $(\mathbf{k}, \mathbf{N}-\mathbf{k})$ is 4 , since the real vector space orthogonal to $\hat{\mathbf{g}}_{\mathbf{k}}$ is two-dimensional and $\hat{\mathbf{u}}_{\mathbf{k}}$ is complex valued.

The equipartition theorem in this case requires that

$$
2 \frac{\rho L^{3}}{2} \frac{4 D_{\mathbf{k}}}{\alpha_{\mathbf{k}}}=\frac{4}{2} k_{B} T
$$

which gives

$$
D_{\mathbf{k}}=\alpha_{\mathbf{k}} \frac{k_{B} T}{2 \rho L^{3}}
$$

when $\mathbf{k} \notin \mathcal{K}$. 
To summarize, the following fluctuation-dissipation relation $(47 ; 70)$ is obtained when considering the constraints 3.25 and 3.22 imposed on the velocity field of the fluid:

$$
D_{\mathbf{k}}= \begin{cases}\frac{k_{B} T}{\rho L^{3}} \alpha_{\mathbf{k}} & , \mathbf{k} \in \mathcal{K} \\ \frac{k_{B} T}{2 \rho L^{3}} \alpha_{\mathbf{k}} & , \mathbf{k} \notin \mathcal{K} .\end{cases}
$$

We remark that the Fourier mode of the fluid associated with $\mathbf{k}=[0,0,0]^{T}$ corresponds to translation of the fluid as a whole. From 3.23 the zero mode has $\alpha_{\mathbf{0}}=0$, which indicates that the fluid has no translational damping. As a consequence of 3.54 the mode $\hat{\mathbf{u}}_{\mathbf{0}}$ is not thermally forced, which can also be understood physically by the conservation of total momentum by the internal thermal fluctuations. Thus for a fluid initially at rest with no net external force on the fluid as a whole, the translational mode remains zero $\hat{\mathbf{u}}_{\mathbf{0}}=\mathbf{0}$ under the thermal forcing.

3.3.4. Numerical Method for the Fluid. To deal with the significant range in time scales for the modes of the fluid and immersed structures, we develop a timestepping scheme that freezes the positions and forces exerted by the elementary particles over a time step $\Delta t$, but otherwise integrates the dynamical equations exactly. With this approximation the set of equations 3.37 can be solved analytically using the methods of stochastic calculus (59). This strategy has similarities to "exponential time differencing" or "exact linear part" numerical methods $(30 ; 36$; 42$)$.

In stochastic differential notation, the fluid equations with both thermal and particle forces can be expressed as

$$
d \hat{\mathbf{u}}_{\mathbf{k}}=-\alpha_{\mathbf{k}} \hat{\mathbf{u}}_{\mathbf{k}} d t+\rho^{-1} \wp_{\mathbf{k}}^{\perp} \hat{\mathbf{f}}_{\mathbf{k}} d t+\sqrt{2 D_{\mathbf{k}}} \wp_{\mathbf{k}}^{\perp} d \tilde{\mathbf{B}}_{\mathbf{k}}(t),
$$

where to simplify the notation the subscript will be dropped for the Fourier modes of the particle force density so that $\hat{\mathbf{f}}_{\mathbf{k}}$ always refers to $\hat{\mathbf{f}}_{\mathrm{prt}, \mathbf{k}}$.

Approximating the particle force as constant over the time interval $\left[t^{\prime}, t\right]$ gives

$$
\begin{aligned}
\hat{\mathbf{u}}_{\mathbf{k}}(t) & =e^{-\alpha_{\mathbf{k}}\left(t-t^{\prime}\right)} \hat{\mathbf{u}}_{\mathbf{k}}\left(t^{\prime}\right)+\frac{1}{\rho \alpha_{\mathbf{k}}}\left(1-e^{-\alpha_{\mathbf{k}}\left(t-t^{\prime}\right)}\right) \wp_{\mathbf{k}}^{\perp} \hat{\mathbf{f}}_{\mathbf{k}}\left(t^{\prime}\right) \\
& +\sqrt{2 D_{\mathbf{k}}} \int_{t^{\prime}}^{t} e^{-\alpha_{\mathbf{k}}(t-s)} \wp_{\mathbf{k}}^{\perp} d \tilde{\mathbf{B}}_{\mathbf{k}}(s),
\end{aligned}
$$

where $\wp_{\mathbf{k}}^{\perp}$ is the projection operation defined in 3.28 and $\int \cdot \wp_{\mathbf{k}}^{\perp} d \tilde{\mathbf{B}}_{\mathbf{k}}(s)$ denotes integration in the sense of Ito (59) over the projected complex-valued Brownian motion $\tilde{\mathbf{B}}_{\mathbf{k}}(t)$ defined in Section 3.3 .3 .

To obtain a numerical scheme for the fluid with finite time step $\Delta t$, each mode is updated at discrete times $n \Delta t$ using the analytic solution 3.56 yielding the stochastic recurrence equation

$$
\hat{\mathbf{u}}_{\mathbf{k}}^{n+1}=e^{-\alpha_{\mathbf{k}} \Delta t} \hat{\mathbf{u}}_{\mathbf{k}}^{n}+\frac{1}{\rho \alpha_{\mathbf{k}}}\left(1-e^{-\alpha_{\mathbf{k}} \Delta t}\right) \wp_{\mathbf{k}}^{\perp} \hat{\mathbf{f}}_{\mathbf{k}}^{n}+\wp_{\mathbf{k}}^{\perp} \hat{\Xi}_{\mathbf{k}}^{n}
$$

where $\hat{\mathbf{u}}_{\mathbf{k}}^{n}=\hat{\mathbf{u}}_{\mathbf{k}}(n \Delta t), \hat{\mathbf{f}}_{\mathbf{k}}^{n}=\hat{\mathbf{f}}_{\mathbf{k}}(n \Delta t)$, and $\hat{\Xi}_{\mathbf{k}}^{n}=\sigma_{\mathbf{k}} \tilde{\eta}_{\mathbf{k}}$.

The notation $\tilde{\eta}_{\mathbf{k}}$ denotes a three dimensional complex-valued random variable, with each real and imaginary component being an independent Gaussian random variable with mean 0 and variance 1 . The random variable $\hat{\Xi}_{\mathbf{k}}^{n}$ accounts for the 
contributions of the stochastic integral in 3.56 over the time step. The variance $\sigma_{\mathbf{k}}^{2}$ can be determined by Ito's Isometry (59) and is given by

$$
\sigma_{\mathbf{k}}^{2}=\frac{D_{\mathbf{k}}}{\alpha_{\mathbf{k}}}\left(1-e^{-2 \alpha_{\mathbf{k}} \Delta t}\right) \text {. }
$$

The constraint 3.25 that ensures the real-valuedness of the velocity field is respected by only applying the update 3.57 to one member of each complex-conjugate pair, and then setting the new value for the partner mode as the complex conjugate of the computed mode. The condition 3.25 also requires that the modes $\hat{\mathbf{u}}_{k}$ with indices $\mathbf{k} \in \mathcal{K}$ have zero imaginary part; this is enforced explicitly in each time step.

3.3.5. Numerical Method for the Immersed Structures. A time-discretization for the equation (3.19) is developed for the advection of the elementary particles by integrating the fluid velocity field over a time step and then averaging the integrated velocity over a spatial neighborhood centered on the old particle position:

$$
\mathbf{X}^{n+1,[j]}-\mathbf{X}^{n,[j]}=\sum_{\mathbf{m}} \delta_{a}\left(\mathbf{x}_{\mathbf{m}}-\mathbf{X}^{n,[j]}\right) \int_{t_{n}}^{t_{n+1}} \mathbf{u}_{\mathbf{m}}(s) d s \Delta x^{3}
$$

where $t_{n}=n \Delta t$ and $\mathbf{X}^{n,[j]}=\mathbf{X}^{[j]}(n \Delta t)$.

A precise integration of the fluid velocity $\mathbf{u}$ is taken which allows for time steps which underresolve the dynamics of some of the Fourier modes of the fluid. This capability is important due to the wide range of time scales that may be associated with the fluid modes and immersed structures in applications. If the time integral is approximated through numerical methods built from (stochastic) Taylor expansions about discrete times, such as Runge-Kutta methods and their stochastic variations (44; 80), then it is important that the method sufficiently resolve the fluctuations of the processes to capture cancellations that occur over time. For instance, if the cancellation is not adequately captured, the numerical value of the integral of velocity will be larger in magnitude than the actual time integrated velocity. For immersed particles, this leads to an overly diffuse behavior where the particles overshoot their correct positions each time step.

From 3.37 the time scale associated with the dynamics of the $\mathbf{k}^{\text {th }}$ mode of the fluid is $1 / \alpha_{\mathbf{k}}$. For the fastest modes of the fluid relevant for the immersed particle dynamics, the above considerations would place a severe restriction on the time step. While there may be clever numerical methods involving (stochastic) Taylor expansions which perform better than anticipated, a different approach will be taken here.

To develop a method that remains accurate for a range of time steps, from those that fully resolve, partially resolve, or completely underresolve the fluid modes, we calculate the time integral in 3.59 by substitution of the analytical expression 3.56 for the Fourier modes of the fluid velocity field. We recall that this approximation only assumes that the elementary particle positions and forces can be considered frozen over a time step. The resulting numerical scheme can therefore be expected to be accurate provided the time step $\Delta t$ is chosen small compared to the time scales of the immersed structures, but with no restriction on the size of the time step relative to the time scales of the fluid modes. We will explain this property more precisely through numerical error analysis in Section 4

In updating the elementary particle positions in the numerical method, the time 
integral in 3.59 will be simulated as a random variable

$$
\begin{gathered}
\boldsymbol{\Gamma}_{\mathbf{m}}^{n}=\int_{t_{n}}^{t_{n+1}} \mathbf{u}_{\mathbf{m}}(s) d s=\sum_{\mathbf{k}} \hat{\boldsymbol{\Gamma}}_{\mathbf{k}}^{n} \exp (i 2 \pi \mathbf{k} \cdot \mathbf{m} / N), \\
\hat{\boldsymbol{\Gamma}}_{\mathbf{k}}^{n}=\int_{t_{n}}^{t_{n+1}} \hat{\mathbf{u}}_{\mathbf{k}}(s) d s,
\end{gathered}
$$

with $\hat{\mathbf{u}}_{k}(s)$ given by (3.56).

By using standard techniques from stochastic calculus, the time integral can be evaluated by defining $\hat{\boldsymbol{\Gamma}}_{\mathbf{k}}^{n}$ to give the Gaussian random variable

$$
\begin{aligned}
\hat{\boldsymbol{\Gamma}}_{\mathbf{k}}^{n} & =-\frac{e^{-\alpha_{\mathbf{k}} \Delta t}-1}{\alpha_{\mathbf{k}}} \hat{\mathbf{u}}_{\mathbf{k}}^{n}+\left(\frac{\Delta t}{\alpha_{\mathbf{k}}}+\left(\frac{1}{\alpha_{\mathbf{k}}}\right)^{2}\left(e^{-\alpha_{\mathbf{k}} \Delta t}-1\right)\right) \rho^{-1} \wp_{\mathbf{k}}^{\perp} \hat{\mathbf{f}}_{\mathbf{k}}^{n} \\
& -\frac{\sqrt{2 D_{\mathbf{k}}}}{\alpha_{\mathbf{k}}} \int_{t_{n}}^{t_{n+1}} e^{-\alpha_{\mathbf{k}}\left(t_{n+1}-r\right)} \wp_{\mathbf{k}}^{\perp} d \tilde{\mathbf{B}}_{\mathbf{k}}(r)+\frac{\sqrt{2 D_{\mathbf{k}}}}{\alpha_{\mathbf{k}}}\left(\wp_{\mathbf{k}}^{\perp} \tilde{\mathbf{B}}_{\mathbf{k}}\left(t_{n+1}\right)-\wp_{\mathbf{k}}^{\perp} \tilde{\mathbf{B}}_{\mathbf{k}}\left(t_{n}\right)\right) .
\end{aligned}
$$

Using 3.56 at times $n \Delta t$ and $(n+1) \Delta t$, this can be expressed more simply as

$$
\begin{aligned}
\hat{\boldsymbol{\Gamma}}_{\mathbf{k}}^{n} & =-\frac{1}{\alpha_{\mathbf{k}}}\left(\hat{\mathbf{u}}_{\mathbf{k}}^{n+1}-\hat{\mathbf{u}}_{\mathbf{k}}^{n}\right)+\rho^{-1} \frac{\wp_{\mathbf{k}}^{\perp} \hat{\mathbf{f}}_{\mathbf{k}}^{n}}{\alpha_{\mathbf{k}}} \Delta t \\
& +\frac{\sqrt{2 D_{\mathbf{k}}}}{\alpha_{\mathbf{k}}}\left(\wp_{\mathbf{k}}^{\perp} \tilde{\mathbf{B}}_{\mathbf{k}}\left(t_{n+1}\right)-\wp_{\mathbf{k}}^{\perp} \tilde{\mathbf{B}}_{\mathbf{k}}\left(t_{n}\right)\right) .
\end{aligned}
$$

The numerical scheme to update the elementary particle positions is then given by

$$
\mathbf{X}^{n+1,[j]}-\mathbf{X}^{n,[j]}=\sum_{\mathbf{m}} \delta_{a}\left(\mathbf{x}_{\mathbf{m}}-\mathbf{X}^{n,[j]}\right) \boldsymbol{\Gamma}_{\mathbf{m}}^{n} \Delta x^{3}
$$

where $\boldsymbol{\Gamma}_{m}^{n}$ is generated each time step. To consistently update the particle positions with the velocity field of the fluid it is required that $\hat{\boldsymbol{\Gamma}}_{\mathbf{k}}^{n}$ be generated with the correct correlations to the fluid modes at the beginning and end of each time step, $\left\{\mathbf{u}_{\mathbf{k}}^{n}\right\}$ and $\left\{\mathbf{u}_{\mathbf{k}}^{n+1}\right\}$. In the next Subsection, a practical approach for doing so is presented.

3.3.6. Method for Generating Modes of the Time Integrated Velocity Field. Since the modes $\hat{\boldsymbol{\Gamma}}_{\mathbf{k}}^{n}$ of the time integrated velocity field and the modes $\hat{\mathbf{u}}_{\mathbf{k}}^{n}$ and $\hat{\mathbf{u}}_{\mathbf{k}}^{n+1}$ of the velocity field evaluated at the beginning and end of a time step are not statistically independent, some care must be taken in generating the corresponding random variables that are used in the simulation. Since $\hat{\boldsymbol{\Gamma}}_{\mathbf{k}}^{n}, \hat{\mathbf{u}}_{\mathbf{k}}^{n}$, and $\hat{\mathbf{u}}_{\mathbf{k}}^{n+1}$ are jointly Gaussian distributed random variables with mean zero, we need only ensure they have the correct covariances between their components. Since the real and imaginary parts of each mode are independent, we shall for clarity consider only the real components with the understanding that the imaginary components are handled in a similar manner.

In deriving a method to generate the time integrated field, it is useful to express the real part $\operatorname{Re}\left(\hat{\boldsymbol{\Gamma}}_{\mathbf{k}}^{n}\right)$ in terms of the following random variables

$$
\operatorname{Re}\left(\hat{\boldsymbol{\Gamma}}_{\mathbf{k}}^{n}\right)=\wp_{\mathbf{k}}^{\perp} \mathbf{A}_{0}+\wp_{\mathbf{k}}^{\perp} \mathbf{A}_{1}+\wp_{\mathbf{k}}^{\perp} \mathbf{A}_{2},
$$


with

$$
\begin{aligned}
\mathbf{A}_{0} & =\frac{1-e^{-\alpha_{\mathbf{k}} \Delta t}}{\alpha_{\mathbf{k}}} \operatorname{Re}\left(\hat{\mathbf{u}}_{\mathbf{k}}^{n}\right)+\left(\frac{\Delta t}{\alpha_{\mathbf{k}}}+\left(\frac{1}{\alpha_{\mathbf{k}}}\right)^{2}\left(e^{-\alpha_{\mathbf{k}} \Delta t}-1\right)\right) \rho^{-1} \operatorname{Re}\left(\hat{\mathbf{f}}_{\mathbf{k}}^{n}\right) \\
\mathbf{A}_{1} & =-\frac{\sqrt{2 D_{\mathbf{k}}}}{\alpha_{\mathbf{k}}} \int_{t_{n}}^{t_{n+1}} e^{-\alpha_{\mathbf{k}}\left(t_{n+1}-s\right)} \operatorname{Re}\left(d \tilde{\mathbf{B}}_{\mathbf{k}}(s)\right) \\
& =-\frac{1}{\alpha_{\mathbf{k}}} \mathbf{\Xi}_{\mathbf{k}}^{n} \\
\mathbf{A}_{2} & =\frac{\sqrt{2 D_{\mathbf{k}}}}{\alpha_{\mathbf{k}}} \operatorname{Re}\left(\tilde{\mathbf{B}}_{\mathbf{k}}\left(t_{n+1}\right)-\tilde{\mathbf{B}}_{\mathbf{k}}\left(t_{n}\right)\right) \\
& =\frac{\sqrt{2 D_{\mathbf{k}}}}{\alpha_{\mathbf{k}}} \int_{t_{n}}^{t_{n+1}} \operatorname{Re}\left(d \tilde{\mathbf{B}}_{\mathbf{k}}(s)\right)
\end{aligned}
$$

The random variables were obtain by reorganizing the terms of 3.62 .

This expression recasts the problem of determining the correlations of $\operatorname{Re}\left(\hat{\boldsymbol{\Gamma}}_{\mathbf{k}}^{n}\right)$ to the problem of determining the correlations of $\mathbf{A}_{0}, \mathbf{A}_{1}$ and $\mathbf{A}_{2}$ with each other and the modes of the fluid velocity. A convenient feature of this approach is that $\mathbf{A}_{0}$ is already determined at the beginning of the time step, and is statistically independent of $\mathbf{A}_{1}$ and $\mathbf{A}_{2}$ by the independent increment property of Brownian motion. This reduces the problem to finding the covariance of $\mathbf{A}_{1}$ and $\mathbf{A}_{2}$. A useful identity for Ito integrals in this context is (59)

$$
E\left(\int_{0}^{t} f(s) d B_{s} \int_{0}^{t} g(r) d B_{r}\right)=\int_{0}^{t} f(s) g(s) d s
$$

where the notation $E(\cdot)$ denotes expectation with respect the underlying Brownian motion (59).

Using 3.69, the covariance is given by

$$
E\left(\mathbf{A}_{1}^{(j)} \mathbf{A}_{2}^{(j)}\right)=-\frac{2 D_{\mathbf{k}}}{\alpha_{\mathbf{k}}^{3}}\left(1-\exp \left(-\alpha_{\mathbf{k}} \Delta t\right)\right),
$$

where the parenthesized superscripts denote the indices of the vector components. When $j \neq j^{\prime}$ the components $\mathbf{A}_{1}^{(j)}$ and $\mathbf{A}_{2}^{\left(j^{\prime}\right)}$ are independent and have zero correlation.

The variance of the components of $\mathbf{A}_{1}$ and $\mathbf{A}_{2}$ are given by

$$
\begin{aligned}
& E\left(\left|\mathbf{A}_{1}^{(j)}\right|^{2}\right)=\frac{D_{\mathbf{k}}}{\alpha_{\mathbf{k}}^{3}}\left(1-\exp \left(-2 \alpha_{\mathbf{k}} \Delta t\right)\right) \\
& E\left(\left|\mathbf{A}_{2}^{(j)}\right|^{2}\right)=\frac{2 D_{\mathbf{k}}}{\alpha_{\mathbf{k}}^{2}} \Delta t
\end{aligned}
$$

From the numerical updating of the fluid variables described in Subsection 3.3.4. $\mathbf{A}_{1}=-\frac{1}{\alpha_{\mathbf{k}}} \boldsymbol{\Xi}_{\mathbf{k}}^{n}$ is already known each time step, so only $\mathbf{A}_{2}$ need be generated. Obtaining this random variable with the correct correlations can be accomplished by generating new standard Gaussian random variables $\boldsymbol{\eta}^{(j)}$ (independent in $j$ with mean 0 and variance 1 ) and by taking the linear combination of the two random variables $\mathbf{A}_{1}^{(j)}$ and $\boldsymbol{\eta}^{(j)}$ given by

$$
\mathbf{A}_{2}^{(j)}=a_{1} \mathbf{A}_{1}^{(j)}+a_{2} \boldsymbol{\eta}^{(j)}
$$


with

$$
a_{1}=\frac{E\left(\mathbf{A}_{1}^{(j)} \mathbf{A}_{2}^{(j)}\right)}{E\left(\left|\mathbf{A}_{1}^{(j)}\right|^{2}\right)}
$$

and

$$
a_{2}=\sqrt{\frac{E\left(\left|\mathbf{A}_{1}^{(j)}\right|^{2}\right) E\left(\left|\mathbf{A}_{2}^{(j)}\right|^{2}\right)-E\left(\mathbf{A}_{1}^{(j)} \mathbf{A}_{2}^{(j)}\right)^{2}}{E\left(\left|\mathbf{A}_{1}^{(j)}\right|^{2}\right)}} .
$$

In this manner, $\operatorname{Re}\left(\hat{\boldsymbol{\Gamma}}_{\mathbf{k}}^{n}\right)$ can be generated from $\mathbf{A}_{0}, \mathbf{A}_{1}$, and $\mathbf{A}_{2}$ with proper accounting of correlations with the modes of the velocity field. The imaginary component $\operatorname{Im}\left(\hat{\boldsymbol{\Gamma}}_{\mathbf{k}}^{n}\right)$ is generated in an analogous manner.

4. Accuracy of the Method. In this section the accuracy of the numerical method is investigated. Three asymptotic scaling regimes of the time step are considered. The first regime applies when the time step is taken sufficiently small to fully resolve the dynamics of the fluid. The second applies when the time step is taken large and completely underresolves the dynamics of the fluid. We finally consider the case in which the time step resolves some but not all of the fluid modes.

Formal error estimates are given which show how the numerical errors scale with respect to the time step and various key parameters. While a rigorous analysis making use of standard stochastic Taylor expansion approaches (44) can be carried out for time steps which are small when compared to the time scales of the fluid and immersed structure dynamics, a completely rigorous analysis of the numerical error when the time step is large and underresolves a subset of the fluid modes is considerably more difficult.

An important feature of the numerical method is the way in which the statistical contributions of the fluid dynamics are taken into account, even when the fluid dynamics are underresolved. As discussed in Subsections 3.3.4 and 3.3.5, the random increments of the elementary particle positions and fluid modes are simulated in such a way that the correct statistics and correlations are preserved over time steps which need only be small compared to the time scales of the immersed structures. While the time step relative to the time scale of the fastest modes of the fluid may be large, this procedure helps keep the local time discretization error small. By contrast, standard finite difference schemes would generally have poor accuracy once the time step exceeded the time scales of the fastest fluid modes.

To quantify the accuracy of the method, the strong error is considered, as defined in $(44)$. Let $\mathbf{X}^{[j]}(t)$ denote the exact solution of equation 3.19 for the elementary particles and $\hat{\mathbf{u}}_{\mathbf{k}}(t)$ denote the exact solution to equation 3.16 for the Fourier modes of the velocity field of the fluid. Let the numerically computed trajectories of the elementary particles be denoted by $\tilde{\mathbf{X}}^{[j]}(t)$ and the numerically computed fluid modes be denoted by $\tilde{\hat{\mathbf{u}}}_{\mathbf{k}}(t)$. Since we shall be interested in the error associated with a typical elementary particle, the superscript $j$ will be dropped throughout the discussion.

The strong error of the numerical method associated with the $\mathbf{k}^{\text {th }}$ mode of the fluid is defined as

$$
\hat{e}_{f l d, \mathbf{k}}(\Delta t)=E\left(\left|\hat{\mathbf{u}}_{\mathbf{k}}(\Delta t)-\tilde{\hat{\mathbf{u}}}_{\mathbf{k}}(\Delta t)\right|\right)
$$


and the strong error associated with an elementary particle is defined as

$$
e_{\mathrm{prt}}(\Delta t)=E(|\mathbf{X}(\Delta t)-\tilde{\mathbf{X}}(\Delta t)|) .
$$

The error associated to the velocity field in physical space is defined as

$$
e_{\mathrm{fld}}(\Delta t)=E\left(\frac{1}{L^{3}} \sum_{\mathbf{m}}\left|\mathbf{u}_{\mathbf{m}}(\Delta t)-\tilde{\mathbf{u}}_{\mathbf{m}}(\Delta t)\right| \Delta x^{3}\right) .
$$

For further discussion of the strong error see (44).

The error expressions above and the estimates given below are intended to characterize the "typical" error for the numerical method; in reality they will of course depend on the particular configuration the elementary particles happen to be in at the beginning of a time step and the details of the forces acting between them. For the purposes of describing the errors incurred in the numerical method's handling of the force interactions, we shall therefore concern ourselves with describing how the errors scale with respect to the various numerical parameters.

In the derivation of the estimates we quantify the error incurred by the numerical method's representation of the stochastic (thermal) components of the structural and fluid dynamics. The estimates presented follow from a systematic formal analysis of the errors resulting from the discretization of the stochastic and deterministic components of the dynamics, including their interaction during a time step. This calculation leads to a uniformly valid expression for time steps sufficiently small that the elementary particles do not move appreciably (relative to their size) during a time step; no assumption is made in the derivation about the magnitude of the time step relative to the time scales of the fluid modes. As the resulting derivations are somewhat technical, we shall in the present paper be content to state the error estimates, discuss their significance, and confirm their validity in a few special cases by numerical simulation. For a detailed derivation see (2).

4.1. Error Estimates for Time Steps which Fully Resolve the Fluid Dynamics. When the time step is taken sufficiently small so that the dynamics of all modes of the fluid are resolved by the stochastic immersed boundary method $\left(\Delta t \ll \min \frac{1}{\alpha_{\mathrm{k}}}\right)$, the following error estimates can be established:

$$
\begin{aligned}
\hat{e}_{\mathrm{fld}, \mathbf{k}}(\Delta t) & \approx \frac{M F^{*}}{\rho} \delta_{a, \mathbf{k}}^{*}\left(\frac{M}{\ell_{F}}+C \frac{1}{a}\right)\left(C^{\prime} v_{\mathrm{frc}}+C^{\prime \prime} v_{\mathrm{thm}}\right) \Delta t^{2} \\
e_{\mathrm{fld}}(\Delta t) & \approx \frac{M F^{*}}{\rho a^{3 / 2} L^{3 / 2}}\left(\frac{M}{\ell_{F}}+C \frac{1}{a}\right)\left(C^{\prime} v_{\mathrm{frc}}+C^{\prime \prime} v_{\mathrm{thm}}\right) \Delta t^{2} \\
e_{\mathrm{prt}}(\Delta t) & \approx\left(Q_{1} v_{\mathrm{thm}}^{2}+C v_{\mathrm{frc}} v_{\mathrm{thm}}+C^{\prime} v_{\mathrm{frc}}^{2}\right) \frac{\Delta t^{2}}{a} \\
& +\frac{M F^{*}}{\rho a^{3}}\left(\frac{M}{\ell_{F}}+C^{\prime \prime} \frac{1}{a}\right)\left(C^{\prime \prime \prime} v_{\mathrm{frc}}+C^{\prime \prime \prime \prime} v_{\mathrm{thm}}\right) \Delta t^{3},
\end{aligned}
$$

where $M$ is the number of elementary particles, $F^{*}$ is the magnitude of the force acting on the elementary particles, and $\ell_{F}$ is the length scale associated with changes in the particle force of order $F^{*}$. It will be assumed throughout that $a \lesssim \ell_{F}$. The 
factor $\hat{\delta}_{a, \mathbf{k}}^{*}$ is the magnitude of the Fourier coefficient for mode $\mathbf{k}$ of the function $\delta_{a}$, averaged over all shifts (see Appendix $\mathrm{B}$ ). We remark that $\hat{\delta}_{a, \mathbf{k}}^{*} \approx 1 / L^{3}$ for $|\mathbf{k}| \ll L / a$, while $\hat{\delta}_{a, \mathbf{k}}^{*}$ decays rapidly for $|\mathbf{k}| \gg L / a$.

In this notation the factors $C$ which are superscripted with primes are approximately independent of the physical parameters, and can be thought of as order unity constants. To avoid cumbersome notation and overly emphasizing the role of these factors the notation is reused in each equation, with the understanding that $C$ denotes distinct factors for each estimate. The subscripted factors $Q$ are also approximately independent of the physical parameters. They are distinguished since numerical values will be estimated for these factors in order to make a comparison between the theoretical estimates and numerical simulations in the case that $F^{*}=0$.

To simplify the expressions the following terms are defined $v_{\mathrm{thm}}=\sqrt{k_{B} T / \rho a^{3}}$ and $v_{\mathrm{frc}}=F^{*} / \mu a$. The factor $v_{\mathrm{thm}}$ can be interpreted via the equipartition theorem of statistical mechanics $(47 ; 70)$ as the velocity scale of thermal fluctuations of an elementary particle of size $a$, since the associated mass will be proportional to $\rho a^{3}$. The term $v_{\text {frc }}$ can be interpreted as the velocity scale associated with the motion of a particle of size $a$ in a viscous fluid when a force of magnitude $F^{*}$ is applied to the particle, since the friction coefficient of a particle is generally proportional to $\mu a$ (15).

The error estimates indicate that the stochastic immersed boundary method has strong first order accuracy as the time step is taken small. An error proportional to $\Delta t^{3}$ is included in $e_{\text {prt }}(\Delta t)$ because its coefficient in certain circumstances can make it comparable to the $\Delta t^{2}$. We remark that the reported proportionality of the errors with respect to $M$, the number of elementary particles, is based on a worst-case scenario where all $M$ particles are clustered near each other. In general the error is expected to scale with a smaller factor reflecting the actual number of particles clustered in a region. Since this depends on details of the force interaction between particles, obtaining a more precise error estimate is technically involved and somewhat application dependent. While in practice the actual numerical error will likely be somewhat better than these factors indicate, we leave further refinements to future work in the context of specific applications.

An important observation is that in the absence of forces on the immersed structures $\left(F^{*}=0\right)$, the fluid modes are simulated exactly (for the reasons discussed in Subsection 3.3.4). Only the elementary particle dynamics incur a temporal discretization error in this case (see Subsection 3.3.5), with the strong error incurred being of first order. A more conventional time stepping scheme based on finite differences would typically incur an error for the velocity mode $\hat{\mathbf{u}}_{\mathbf{k}}$ which includes a contribution which scales as $C N^{-3 / 2} v_{\text {thm }}\left(\alpha_{\mathbf{k}} \Delta t\right)^{n+1 / 2}$ for some integer $n$. Such an error fails to remain small compared to the actual velocity change over a time step as soon as $\Delta t \gtrsim 1 / \alpha_{\mathbf{k}}$. For the numerical method developed in Section 3 the exact representation of the stochastic fluid dynamics, apart from the response to the forces exerted by the immersed structures, maintains better accuracy even as the time step underresolves the fluid dynamics.

As demonstrated in Figure 4.1 the theoretical error estimate for the elementary particles agrees well with numerical simulations in the absence of particle forces $\left(F^{*}=0\right)$. The numerical results were obtained from simulations of the fluid-particle system with physical parameters in Table 4.2. In the comparison, the factors $Q$ were computed from theoretical expressions arising in the derivation of the estimates, and their numerical values are given in Appendix D, It should be emphasized that the error estimates are stated as formal approximations, not as upper bounds. In the case 
that there are particle forces, the discretization error depends on a number of details of the force structure, and therefore numerical comparison with simulations is left to future work in the context of specific applications.

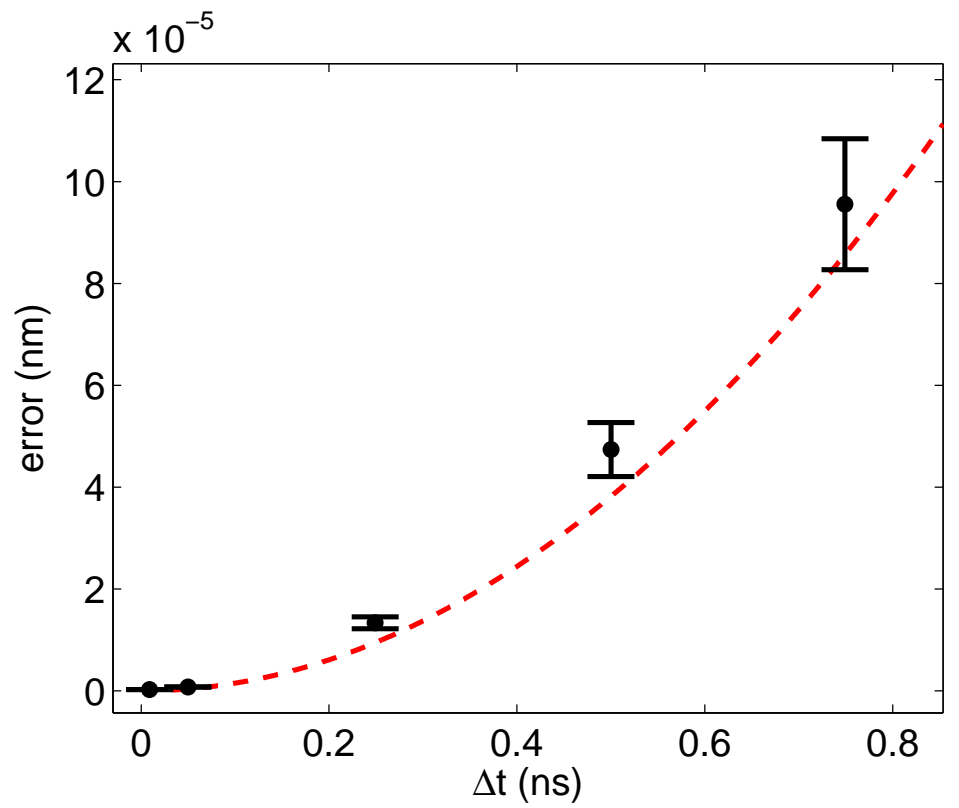

FIG. 4.1. A comparison of the analytic error estimate for the elementary particle positions $e_{p r t}(\Delta t)$ given in equation 4.6 with a numerical estimation of the error for the small to intermediate time step regime $\Delta t \ll \rho a^{2} / \mu$ with $F^{*}=0$ and parameter values given in Table 4.2. The dashed line denotes $e_{\text {prt }}(\Delta t)$. The data points denote the error as estimated from numerical simulations using the method. The error bars indicate one standard deviation of the sampled values. To compute the numerical estimates of the error, simulations were performed with each given time step $\Delta t$ and compared with an ensemble of reference trajectories obtained from a high resolution simulation sufficient to resolve the dynamics of all $N^{3}$ modes of the fluid. The high resolution simulation had a time step of $\Delta t=10^{-3} n s<\min 1 / \alpha_{\mathbf{k}}$, where $\min 1 / \alpha_{\mathbf{k}}=3.94 \times 10^{-2} n s$ for the parameter values given in Table 4.2. From the results, we see that the estimate given in equation 4.6 quantifies the error well for $\Delta t \ll \rho a^{2} / \mu=0.976$ ns.

\subsection{Error Estimates for Time Steps which Underresolve All Fluid} Modes. We now present estimates for the error of the numerical method when the time step is taken large enough to underresolve all modes of the fluid, but always small enough to resolve the elementary particle dynamics:

$$
\max \frac{1}{\alpha_{\mathbf{k}}} \ll \Delta t \ll \tau_{\operatorname{mov}}(a) .
$$

The notation $\tau_{\operatorname{mov}}(a)$ denotes the time required for an elementary particle to move a displacement equal to its size $a$ either by advection or diffusion. In this regime the following error estimates can be established:

$$
\hat{e}_{\mathrm{fld}, \mathbf{k}}(\Delta t) \approx \frac{M F^{*} L^{2}}{\mu|\mathbf{k}|^{2}} \delta_{a, \mathbf{k}}^{*}\left(\frac{M}{\ell_{F}}+C \frac{1}{a}\right)\left(C^{\prime} v_{\mathrm{frc}} \Delta t+C^{\prime \prime} \sqrt{D} \Delta t^{1 / 2}\right)
$$




$$
e_{\mathrm{fld}}(\Delta t) \approx \sqrt{\frac{a}{L}} \frac{M F^{*}}{\mu L}\left(\frac{M}{\ell_{F}}+C \frac{1}{a}\right)\left(C^{\prime} v_{\mathrm{frc}} \Delta t+C^{\prime \prime} \sqrt{D} \Delta t^{1 / 2}\right)
$$

$$
\begin{aligned}
e_{\mathrm{prt}}(\Delta t) & \approx Q_{2} \frac{D}{a} \Delta t \\
& +\left(C \frac{M}{\ell_{F}}+C^{\prime} \frac{1}{a}\right)\left(\sqrt{D} v_{\mathrm{frc}} \Delta t^{3 / 2}+v_{\mathrm{frc}}^{2} \Delta t^{2}\right),
\end{aligned}
$$

where $D$ denotes the diffusion coefficient of an immersed particle (see Section 5.1) and the factors $C$ and $Q$ denote order unity nondimensional constants as discussed in Subsection 4.1. The other terms are the same as in Subsection 4.1.

The smaller powers of $\Delta t$ appearing in the error estimates may suggest that the accuracy is deteriorating more rapidly with respect to the time step in the underresolved regime under discussion, but in fact the opposite is true. The error estimates reported above are in fact, for the range of time steps defining the underresolved regime, considerably smaller than the extrapolation of the error estimates in Subsection 4.1 which are valid only for the fully resolved regime. Indeed, the ratio of terms appearing in the above estimates to corresponding terms in the equations in Subsection 4.1 involve ratios such as $\rho L^{2} /\left(\mu|\mathbf{k}|^{2} \Delta t\right), D^{1 / 2} /\left(v_{\text {thm }} \Delta t^{1 / 2}\right)$, and $L^{1 / 2} a^{3 / 2} \rho / \mu \Delta t$, all of which are much smaller than one in the asymptotic regime 4.7

A more important point is that the numerical errors remain small relative to the changes in the system variables throughout this range of time steps, so that the numerical method maintains accuracy for all $\Delta t \lesssim \tau_{\operatorname{mov}}(a)$. This can be seen by observing that the changes in the system variables over a time step falling in the regime 4.7 can be estimated as

$$
\begin{aligned}
\left|\delta \hat{\mathbf{u}}_{\mathbf{k}}\right| & \approx C \frac{M F^{*} \delta_{a, k}^{*}}{\rho \alpha_{\mathbf{k}}}+C^{\prime} \frac{v_{\mathrm{thm}}}{N^{3 / 2}}, \\
|\delta \mathbf{u}| & \approx C v_{\mathrm{frc}}+C^{\prime} v_{\mathrm{thm}} \\
|\delta \mathbf{X}| & \approx C \sqrt{D \Delta t}+C^{\prime} v_{\mathrm{frc}} \Delta t
\end{aligned}
$$

where the factors $C$ denote order unity nondimensional constants as discussed in Subsection 4.1 The notation $|\delta[\cdot]|$ indicates the absolute value of an increment of a variable over the time step.

Since the velocity field of the fluid is completely underresolved, it changes by an amount comparable to its equilibrium value independently of the size of the time step. The ratios of the error estimates to the corresponding true changes in the system variables in 4.13 can be bounded by sums and products of the nondimensional groups $\sqrt{D \Delta t} / a, v_{\text {frc }} \Delta t / a, M \frac{a}{L}$, and $M \frac{a}{\ell_{F}}$. The former two nondimensional groups involving the time step are both small by definition of the constraint $\Delta t \ll \tau_{\operatorname{mov}}(a)$ determining the asymptotic regime 4.7 under consideration. The nondimensional parameters $M \frac{a}{L}$ and $M \frac{a}{\ell_{F}}$ will be order unity or smaller when the system involves a small number of elementary particles. When the system contains a large number of elementary particles, these nondimensional groups can become large and the error 
estimates become worse. While it is certainly to be expected that the presence of more complex structures involving more elementary particles will generally incur more error in the numerical simulation, we stress that the scaling of our errors with large $M$ are surely too pessimistic. We therefore do not lay undue emphasis on the behavior of the errors for large $M$, which in any event will depend heavily on the details of the force structure.

We emphasize that unlike traditional numerical analysis the presence of terms proportional to $\Delta t$ in the error estimate 4.10 does not imply that the method is inconsistent. It must be remembered that these error estimates are appropriate not in the $\Delta t \downarrow 0$ limit, but rather in the asymptotic regime4.7 A more careful consideration of the sizes of the errors relative to the true changes in the system variables over a time step shows that our numerical method does in fact remain accurate for all time steps $\Delta t \ll \tau_{\text {mov }}(a)$, even if the fluid modes are underresolved. Vital to this result was the use of the stochastic integral formula 3.56 for the action of the thermal forces on the velocity field of the fluid, and the systematic consideration in Subsection 3.3.5 of how to correlate the stochastic component of the velocity field of the fluid with the random motion of the immersed structures. Without these developments, the resulting numerical method could not be expected to have good accuracy for time steps in the regime 4.7

In Figure 4.2, the theoretical error estimate 4.10 for the elementary particle positions over a long time step is compared with the results of a numerical simulation in the case that there are no particle forces $\left(F^{*}=0\right)$. The numerical results were obtained from simulations of the fluid-particle system with physical parameters in Table 4.2. In the comparison, the factors $Q$ were computed from the theoretical analysis with values given in Appendix D. The numerical simulations show good quantitative agreement with the formal error estimate 4.10, We remark that the estimate is to be understood as an approximation and not a rigorous upper bound. This agreement is evidence of the validity of the formal analysis. As discussed in Section 4.1, the errors arising in the presence of forces are not as explicitly quantifiable. We leave further discussion and verification of the estimates to future work in the context of specific applications.

4.3. Error Estimates for Time Steps which Underresolve Only Some Fluid Modes. A key feature of the stochastic numerical scheme proposed in this work is that time steps can be chosen which only partially resolve the fluid dynamics. That is, the method need neither resolve all of the velocity modes nor completely neglect the inertia of the velocity field (as in Brownian/Stokesian dynamics (15; 22; 75; 77)). Rather, the time step can be chosen as needed to resolve the appropriate degrees of freedom of the fluid-particle system, having the fluid and thermal fluctuations interact appropriately with the structures. The case is now discussed in which the time step $\Delta t$ falls within the intermediate regime

$$
\min \frac{1}{\alpha_{\mathbf{k}}} \lesssim \Delta t \lesssim \max \frac{1}{\alpha_{\mathbf{k}}}
$$

where the dynamics of the fluid is only partially resolved. It turns out that the error estimates for the fully resolved regime (Subsection 4.1) and the underresolved regime (Subsection 4.2) each serve separately as formal upper bounds for all time steps, including the intermediate regime 4.14. Intuitively, then, one expects the numerical method to behave accurately over this intermediate range of time scales as well. To provide more quantitative support for this statement, error estimates are developed 


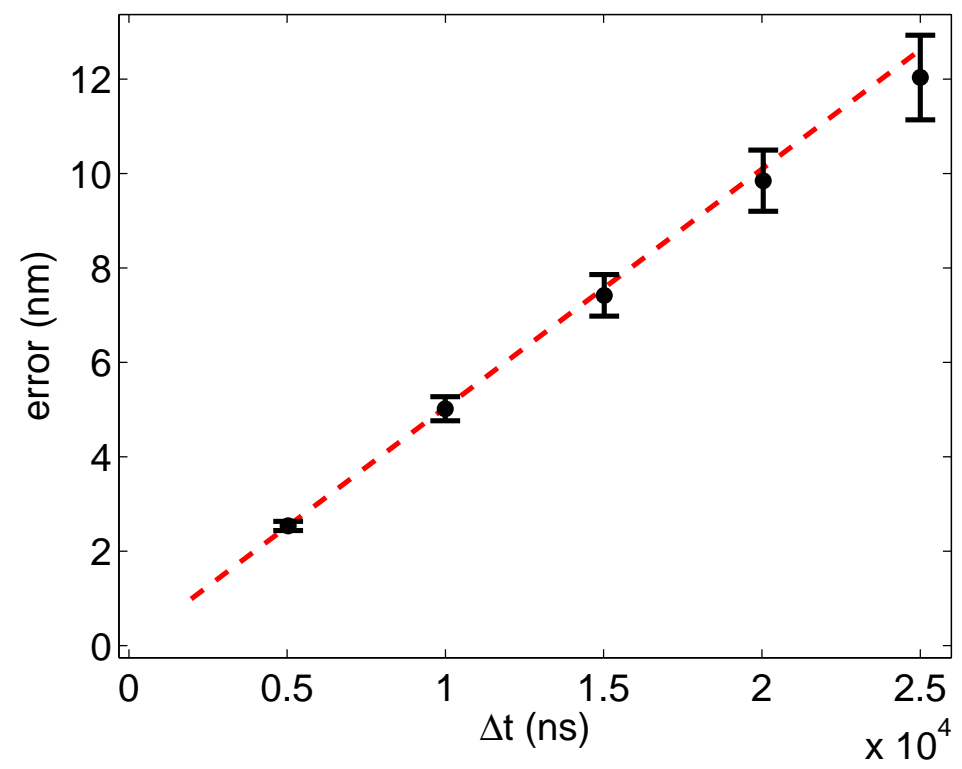

FIG. 4.2. A comparison of the analytic error estimate for the elementary particle positions $e_{p r t}(\Delta t)$ given in equation 4.10 with a numerical estimation of the error for the large time step regime $\max 1 / \alpha_{\mathbf{k}} \ll \Delta t \ll \tau_{\text {mov }}$ with $F^{*}=0$ and parameter values given in Table 4.2. The dashed line denotes $e_{\text {prt }}(\Delta t)$. The data points denote the error as estimated from numerical simulations using the method. The error bars indicate one standard deviation of the sampled values. To compute the numerical estimates of the error, simulations were performed with each given time step $\Delta t$ and compared with an ensemble of reference trajectories obtained from a high resolution simulation with a time step of $\Delta t=1.0 n s<\max 1 / \alpha_{\mathbf{k}}$ where $\max 1 / \alpha_{\mathbf{k}}=25.4 n$ s for the parameter values given in Table 4.2. From the results, we see that the estimate given in equation 4.10 quantifies the error well for $\max 1 / \alpha_{\mathbf{k}} \ll \Delta t \ll \tau_{\text {mov }}$, where $\tau_{\text {mov }} \approx a^{2} / D=1.95 \times 10^{5} \mathrm{~ns}$.

for the asymptotic regime

$$
\frac{\rho a^{2}}{\mu} \ll \Delta t \ll \max \frac{1}{\alpha_{\mathbf{k}}} .
$$

Then, for fluid modes that are well resolved, we have

$$
\hat{e}_{\mathrm{fld}, \mathbf{k}}(\Delta t) \approx \frac{M F^{*}}{\rho} \delta_{a, \mathbf{k}}^{*}\left(\frac{M}{\ell_{F}}+C \frac{1}{a}\right)\left(C^{\prime} v_{\mathrm{frc}} \Delta t^{2}+C^{\prime \prime} \sqrt{D} \Delta t^{3 / 2}\right) \quad \text { if } \alpha_{\mathbf{k}} \Delta t \ll 1,
$$

while the underresolved modes (with $\alpha_{\mathbf{k}} \Delta t \gg 1$ ) have the same error estimate 4.8 as in the fully underresolved case.

The errors incurred in the physical space variables describing the velocity and elementary particle positions can, in the asymptotic regime 4.15, be estimated as

$$
e_{\mathrm{fld}}(\Delta t) \approx \frac{M F^{*}}{\rho \nu^{3 / 4} L^{3 / 2}}\left(\frac{M}{\ell_{F}}+C \frac{1}{a}\right)\left(C^{\prime} v_{\mathrm{frc}} \Delta t^{5 / 4}+C^{\prime \prime} \sqrt{D} \Delta t^{3 / 4}\right)
$$




$$
\begin{aligned}
e_{\mathrm{prt}}(\Delta t) & \approx Q_{2} \frac{D}{a} \Delta t \\
& +\left(\frac{M}{\ell_{F}}+C^{\prime} \frac{1}{a}\right)\left(\sqrt{D} v_{\mathrm{frc}} \Delta t^{3 / 2}+v_{\mathrm{frc}}^{2} \Delta t^{2}\right),
\end{aligned}
$$

where $\nu=\mu / \rho$.

These errors can be compared with the size of the actual changes in the system variables over a time step in the regime 4.15, which can be estimated by the same formulas as 4.13 except that the resolved velocity modes have changes of approximate size

$$
\left|\delta \hat{\mathbf{u}}_{\mathbf{k}}\right| \approx C \frac{M F^{*} \delta_{a, k}^{*}}{\rho} \Delta t+C^{\prime} \frac{v_{\mathrm{thm}} \sqrt{\alpha_{\mathbf{k}} \Delta t}}{N^{3 / 2}}
$$

The ratio of the errors to the corresponding magnitudes of the actual changes of the system variables over a time step is controlled by sums and products of the nondimensional quantities $\sqrt{D \Delta t} / a, v_{\mathrm{frc}} \Delta t / a,(\nu \Delta t)^{1 / 4} / L^{1 / 2}, M \frac{a}{L}$, and $\frac{M a}{\ell_{F}}$. The former three remain small in the asymptotic regime 4.15 under consideration, while the last two nondimensional groups (independent of time step) are related to our somewhat pessimistic bound on the force errors, as discussed in Subsection 4.1. The numerical method is thereby shown to remain theoretically accurate within this intermediate asymptotic regime. In the absence of particle forces $\left(F^{*}=0\right)$, the error estimates become identical to those for the unresolved fluid regime (Subsection 4.2).

One could also study the intermediate asymptotic regime

$$
\min \frac{1}{\alpha_{\mathbf{k}}} \ll \Delta t \ll \frac{\rho a^{2}}{\mu}
$$

which exists only when $\Delta x \ll a$. For these time steps, all error estimates presented in Subsection 4.1 for the fully resolved regime remain valid, except that the estimate for the individual underresolved fluid modes is altered to

$$
\hat{e}_{\mathrm{fld}, \mathbf{k}}(\Delta t) \approx \frac{M F^{*} L^{2}}{\mu|\mathbf{k}|^{2}}\left(\frac{M}{\ell_{F}}+C \frac{1}{a}\right) \delta_{a, \mathbf{k}}^{*}\left(C^{\prime} v_{\mathrm{frc}}+C^{\prime \prime} v_{\mathrm{thm}}\right) \Delta t .
$$

As with the other regimes, the errors in this regime are small relative to the magnitude of the changes of the actual system variables over a time step.

By simple extension of the above arguments for time steps falling at the transitions between the asymptotic regimes, we see that the numerical method has been designed to remain theoretically accurate for all time steps $\Delta t \ll \tau_{\operatorname{mov}}(a)$, regardless of how well the fluid dynamics are resolved.

5. Physical Behavior of the Method and Numerical Results. To ensure that the immersed boundary method with thermal fluctuations serves as a plausible physical framework for modeling microscale systems, we verify that the method exhibits several fundamental features which are correct according to the laws of statistical physics (70). In Subsection 5.1 an expression for the diffusion coefficient of immersed particles is derived, and it is shown that in three dimensions the mean squared displacement scales linearly in time and inversely in the particle size. It is further shown in Subsection 5.2 that the stochastic immersed boundary method captures the correct $\tau^{-3 / 2}$ power law for the decay of the tail of the autocorrelation function of the particle velocity $(6 ; 16 ; 20 ; 23 ; 37 ; 38 ; 54 ; 67)$. 
In determining the thermal forcing in Subsection 3.3.3 we imposed the requirement that the degrees of freedom of the fluid obey Boltzmann statistics in thermal equilibrium. In fact, the complete system including immersed structures should obey Boltzmann statistics. In Subsection [5.3 we study the equilibrium statistics of immersed particles subject to a conservative force and show through numerical simulation that they do exhibit the correct Boltzmann statistics. To demonstrate some applications and as a further verification of the physical plausibility of the method, it is shown in Subsections [5.4-5.6 how the method can be used to model osmotic effects such as the pressure of confined particles, dimers, and polymers $(21 ; 61)$. In Subsection [5.7 another application to a basic model of a molecular motor protein immersed in a fluid subjected to a hydrodynamic load force is presented (66).

5.1. Diffusion of Immersed Particles. In this section the diffusion of particles in the stochastic immersed boundary method is discussed and an expression for the diffusion coefficient is derived. As part of the analysis it is shown that the correct diffusive scaling is obtained for three dimensional systems. To verify the validity of the approximations made in the analysis and to demonstrate the applicability of these results in practice, the results of the analysis are compared to the results of numerical simulations.

In three dimensions, the diffusion coefficient for a single particle is defined as

$$
D=\lim _{t \rightarrow \infty} \frac{\left\langle|\mathbf{X}(t)-\mathbf{X}(0)|^{2}\right\rangle}{6 t} .
$$

In the notation the superscripts on the particle position are suppressed since only a single immersed particle is considered.

An estimate for the diffusion coefficient of a single particle (with no interactions with other particles) in the stochastic immersed boundary method is derived in Subsection 5.1.1 from the autocorrelation function of the velocity field of the fluid. This estimate can be expressed as

$$
D=\frac{k_{B} T L^{3}}{3 \rho} \sum_{\mathbf{k}} \frac{\left|\hat{\delta}_{a, \mathbf{k}}\right|^{2} \Upsilon_{\mathbf{k}}}{\alpha_{\mathbf{k}}}
$$

where $\Upsilon_{\mathbf{k}}$ is defined in appendix $\mathrm{C}$ and $\hat{\delta}_{a, \mathbf{k}}$ is defined in appendix $\mathrm{B}$. This diffusivity as simulated by the stochastic immersed boundary method exhibits the physically correct scaling with respect to physical parameters (45).

The diffusion coefficient is estimated from the numerical simulations using

$$
\tilde{D} \approx \frac{1}{6 n t_{1}} \sum_{m=1}^{n}\left|\tilde{\mathbf{X}}^{m}\left(t_{1}\right)-\tilde{\mathbf{X}}^{m}(0)\right|^{2},
$$

where $n$ is the number of sampled trajectories of fixed duration $t_{1}$. The notation $\tilde{\mathbf{X}}^{m}$ denotes the simulated particle position from the $m^{\text {th }}$ trajectory.

In Figure 5.1, the theoretical estimate of the diffusion coefficient as given in equation 5.2 is compared to the numerical estimate given in equation 5.3 for particles with sizes $a=1,2,3,4,5$ and for a long time step which underresolves the dynamics of the fluid. For the parameters of the fluid-particle system used in the numerical simulations, see table 4.2. For each particle size, the numerical estimates were made from $n=10^{4}$ sampled trajectories with $\Delta t=10^{3} \mathrm{~ns}$ and $t_{1}=10^{4} \mathrm{~ns}$. We remark that in the simulations, while the time step underresolves the fastest modes of the fluid, 


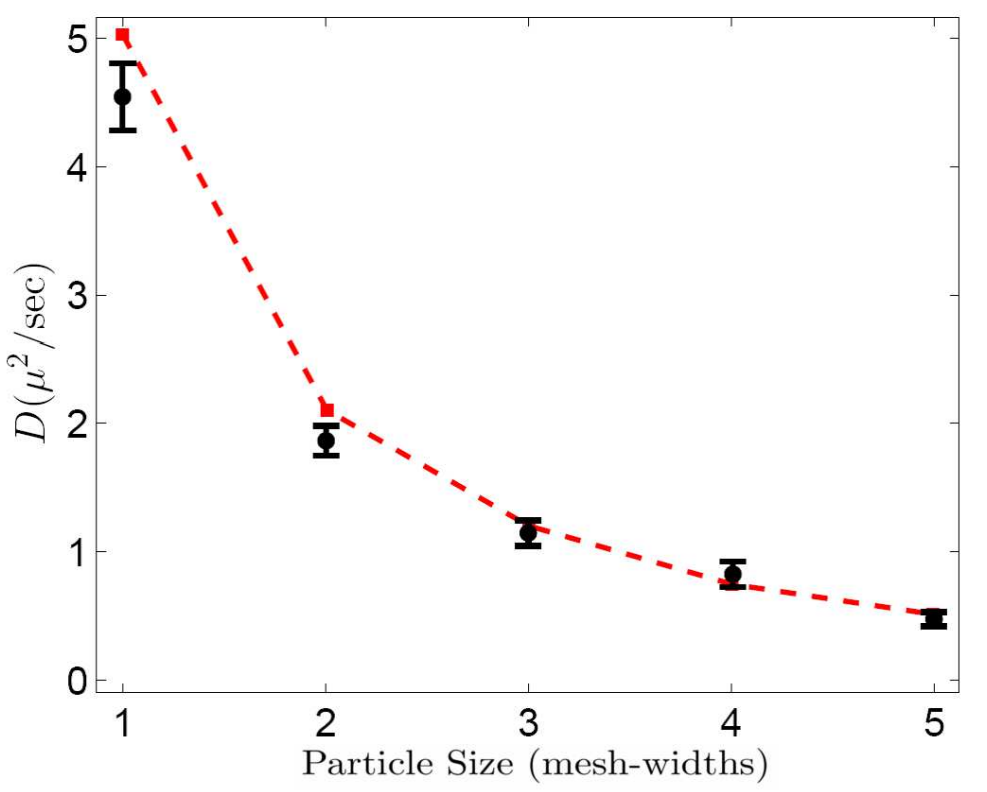

FIG. 5.1. A comparison of the analytic estimate 5.2 for particles of the IB method with numerically estimated diffusion coefficients for the parameters given in Table 4.2. The dashed line indicates the analytic estimate 5.2. The data points denote estimates of the diffusion coefficient obtained from numerical simulations using the method. The error bars denote one standard deviation of the sampled values. In the numerical simulations long time steps satisfying $\max 1 / \alpha_{\mathbf{k}} \ll \Delta t$ were taken which under For the parameters values given in Table 4.2 the time step $\Delta t=10^{3}$ ns was used in the numerical simulations, where $\max 1 / \alpha_{\mathbf{k}}=25.4 \mathrm{~ns}$. The particle size $k_{0}$ corresponds to the parameter value $a=k_{0} \Delta x$ of the particle representation function $\delta_{a}$ defined in appendix $\$$, where $k_{0}$ controls the number of mesh-widths spanned by the $\delta_{a}$ function.

there is still good agreement between the diffusion coefficient of the simulated particle and the theoretical estimate 5.2. A tabulation of the theoretical diffusion coefficient as given in equation 5.2 for the immersed boundary method with various ratios of $a / \Delta x$ and $a / L$ can be found in (45)).

5.1.1. Derivation of the Diffusion Coefficient. To derive an analytic estimate for the diffusion coefficient the semidiscretized equations 3.16 and 3.18 are considered. Alternatively, the diffusion coefficient can also be derived directly from the stochastic immersed boundary equations by a stochastic mode reduction procedure (46). In equations 3.16 and 3.18 the immersed particle dynamics are given by

$$
\frac{d \mathbf{X}(t)}{d t}=\mathbf{U}(\mathbf{X}(t), t),
$$

where $\mathbf{U}$ is given in Fourier space by

$$
\mathbf{U}(\mathbf{x}, t)=\sum_{\mathbf{k}} L^{3} \hat{\delta}_{a, \mathbf{k}}(\mathbf{x}) \hat{\mathbf{u}}_{\mathbf{k}}(t) \exp (i 2 \pi \mathbf{x} \cdot \mathbf{k} / L),
$$

with the coefficient $\hat{\delta}_{a, \mathbf{k}}(\mathbf{x})$ defined in appendix B,

The autocorrelation function of the velocity of an immersed particle is

$$
R(t, t+\tau)=\langle\mathbf{U}(\mathbf{X}(t), t) \cdot \mathbf{U}(\mathbf{X}(t+\tau), t+\tau)\rangle .
$$


To estimate this, we use the fact that the statistics of $\mathbf{U}(\mathbf{x}, t)$ are approximately shift invariant in both $t$ and $\mathbf{x}$, with the approximation improving as the spatial grid is refined. We further assume that the time scale associated with the fluid velocity is small relative to the time scale of the immersed particle motion, so that a particle moves a negligible distance relative to its size $a$ during the correlation time of the fluid velocity. These approximations give

$$
R(t, t+\tau) \approx\langle\mathbf{U}(0,0) \cdot \mathbf{U}(0, \tau)\rangle:=R(\tau) .
$$

By applying (C.5), the autocorrelation function of the velocity of an immersed particle can then be expressed as

$$
\begin{aligned}
R(\tau) & \approx \sum_{\mathbf{k}, \mathbf{k}^{\prime}} L^{6} \overline{\hat{\delta}}_{a, \mathbf{k}} \hat{\delta}_{a, \mathbf{k}^{\prime}}\left\langle\overline{\mathbf{u}_{\mathbf{k}}}(t) \cdot \mathbf{u}_{\mathbf{k}^{\prime}}(t+\tau)\right\rangle \\
& =\sum_{\mathbf{k} \in K} L^{6}\left|\hat{\delta}_{a, \mathbf{k}}\right|^{2} 3 \frac{D_{k}}{\alpha_{\mathbf{k}}} e^{-\alpha_{\mathbf{k}}|\tau|} \\
& +\sum_{\mathbf{k} \notin K} L^{6}\left|\hat{\delta}_{a, \mathbf{k}}\right|^{2} 4 \frac{D_{k}}{\alpha_{\mathbf{k}}} e^{-\alpha_{\mathbf{k}}|\tau|} \\
& =\frac{k_{B} T L^{3}}{\rho} \sum_{\mathbf{k}}\left|\hat{\delta}_{a, \mathbf{k}}\right|^{2} \Upsilon_{\mathbf{k}} e^{-\alpha_{\mathbf{k}}|\tau|},
\end{aligned}
$$

where $\Upsilon_{\mathbf{k}}$ is defined in appendix $\mathrm{C}$

An important point for the numerical method developed in Section 3 is that this structure of the correlation function is preserved even for finite time steps, provided only that the time step is small enough that the immersed elementary particles do not move significantly during a time step $\left(\Delta t \ll \tau_{\text {diff }}(a)\right)$, where $\tau_{\text {diff }}(a)$ is the time scale of a particle to diffuse over a distance equal to its size $a$. Were we to have used instead a numerical method based on a stochastic Taylor expansion (44), we would have to restrict the time step $\Delta t$ to be small enough so that $R(\tau)$ is well approximated by a Taylor expansion for $|\tau| \lesssim \Delta t$, which would add the additional restriction that $\Delta t \ll 1 / \alpha_{\mathbf{k}}$. Our more accurate representation for the fluid dynamics over a time step allows us to obviate this other condition, as demonstrated in Figure 5.1 .

A useful identity relating the autocorrelation function to the mean squared displacement of an immersed particle is

$$
\begin{aligned}
\left\langle|\mathbf{X}(t)-\mathbf{X}(0)|^{2}\right\rangle & =\left\langle\int_{0}^{t} \frac{d \mathbf{X}(s)}{d s} d s \cdot \int_{0}^{t} \frac{d \mathbf{X}(r)}{d r} d r\right\rangle \\
& =2 \int_{0}^{t} R(r) \cdot(t-r) d r .
\end{aligned}
$$

This allows for the diffusion coefficient to be estimated by the Kubo formula (47):

$$
D=\frac{1}{3} \int_{0}^{\infty} R(r) d r
$$

By substituting the estimate 5.8 into 5.10 and evaluating the integral, the expression 5.2 for the diffusion coefficient is obtained. 
5.2. Algebraic Decay of Velocity Autocorrelation Function. For immersed particles diffusing in a viscous fluid, the particle motion is strongly coupled to the motion of the fluid. As a particle moves along a particular direction, fluid is dragged along with it. When the particle changes direction, it is resisted by a viscous force arising from its motion relative to the nearby fluid with momentum related to the recent past of the particle's motion. This induces a somewhat stronger memory in the particle velocity than a standard model based on a constant Stokes drag would predict. In particular, a careful analysis of physical Brownian motion, including a more detailed model for the force between an immersed particle and the surrounding fluid, yields for $D \ll \mu / \rho \quad(6 ; 16 ; 20 ; 23 ; 37 ; 38 ; 54 ; 67 ; 78)$

$$
R(\tau) \approx \frac{k_{B} T \rho^{1 / 2}}{4 \mu^{3 / 2}} \tau^{-3 / 2} \text { for } \tau \gg \rho a^{2} / \mu .
$$

The condition $D \ll \mu / \rho$ can readily be checked to hold for typical microbiological systems.

For the stochastic immersed boundary method, it is shown in Subsection 5.2.1 that this general behavior is recovered with

$$
R(\tau) \approx\left[C_{\mathrm{IB}} \frac{k_{B} T \rho^{1 / 2}}{\mu^{3 / 2}}\right] \tau^{-3 / 2} \text { for } \rho a^{2} / \mu \ll \tau \ll \rho L^{2} / \mu,
$$

where $C_{\mathrm{IB}}=\frac{1}{4 \pi^{3 / 2}}$. The constant prefactors differ slightly due to the different ways particles are represented in the physical model and immersed boundary method.

The restriction that $\tau \ll \rho L^{2} / \mu$ for the $\tau^{-3 / 2}$ scaling in the immersed boundary method is a finite size effect which should have an analogue for physical Brownian motion. For very long times where $\tau \gg \rho L^{2} / \mu$ the correlation function $R(\tau)$ decays exponentially, with rate governed by that of the lowest wavenumber modes in the Fourier series (5.8). However, by these times the autocorrelation function would already be very small so this very long time regime is of little practical interest.

These results show that the decay of the particle velocity autocorrelation function in the stochastic immersed boundary method has the correct scaling with respect to time and physical parameters.

5.2.1. Derivation of Algebraic Decay of Velocity Autocorrelation Function. In this discussion, the reference to wavenumbers $\mathbf{k}$ implicitly indicates the value within the equivalence class of aliased wavenumbers such that each component $\left|\mathbf{k}^{(j)}\right| \leq N / 2$. For this purpose one can choose any scheme to select a unique value when $\mathbf{k}$ lies on the boundary of this set.

First observe from the scaling properties of Fourier transforms and the definition of $\delta_{a}$ from (A.2) that

$$
\hat{\delta}_{a, \mathbf{k}} \approx \hat{\delta}_{a, \mathbf{0}}=\frac{1}{L^{3}} \text { for }|\mathbf{k}| \ll L / a,
$$

and $\hat{\delta}_{a, \mathbf{k}}$ decays rapidly with respect to $|\mathbf{k}| a / L$. Along with the fact that the high wavenumber components of $R(\tau)$ decay at a faster rate $\alpha_{\mathbf{k}}$ than the low wavenumber components, it then follows from the Fourier series representation 5.8 for the particle velocity autocorrelation function that the sum will be dominated by the terms with $|\mathbf{k}| \lesssim L / a$

Over the intermediate asymptotic time interval indicated in 5.12 , the time $t$ is small compared to the decay time $1 / \alpha_{\mathbf{k}} \sim \rho L^{2} / \mu$ of the low wavenumber modes 
$|\mathbf{k}| \sim 1$, but large compared to the decay time $1 / \alpha_{\mathbf{k}} \sim \rho a^{2} / \mu$ of the (relatively high) wavenumber modes $|\mathbf{k}| \sim L / a$ corresponding to the length scale of the particle. Combining these observations, there exists a time-dependent wavenumber scale $k_{c}(t)$ which satisfies $1 \ll k_{c}(t) \ll N / 2$ such that $e^{-\alpha_{\mathbf{k}} t} \approx 1$ for $|\mathbf{k}| \ll k_{c}(t)$ and $e^{-\alpha_{\mathbf{k}}(t)} \approx 0$ for wavenumbers such that $|\mathbf{k}| \gg k_{c}(t)$. Consequently, over the intermediate asymptotic time interval, the Fourier series 5.8 is dominated by contributions from wavenumbers $1 \leq|\mathbf{k}| \lesssim k_{c}(t) \ll N / 2$. These observations allow us to make the following simplifying approximations over the time interval $\rho a^{2} / \mu \ll t \ll \rho L^{2} / \mu$ :

- The prefactors multiplying the exponential in each Fourier series term may be approximated by their low wavenumber limits:

$$
\left|\hat{\delta}_{a, \mathbf{k}}\right|^{2} \Upsilon_{\mathbf{k}} \approx 2 / L^{6} \text { for }|\mathbf{k}| \ll N / 2 .
$$

- The decay rate in the exponential may be approximated for $|\mathbf{k}| \ll N / 2$ by its low wavenumber asymptotics

$$
\alpha_{\mathbf{k}} \approx A|\mathbf{k}|^{2} ; A=4 \pi^{2} \mu \rho^{-1} L^{-2} .
$$

- The Fourier sum may be extended to the full integer lattice, because with the replacement 5.15, the additional terms for large wavenumbers will be exponentially small and make a negligible contribution.

- This Fourier sum over the integer lattice can be approximated by an integral over continuous $\mathbf{k}$, because the dominant contribution comes from a large number of lattice sites $1 \leq|\mathbf{k}| \lesssim k_{c}(t)$, with $k_{c}(t) \gg 1$.

Applying these simplifications and then changing to spherical coordinates with radial variable $k=|\mathbf{k}|$, we obtain

$$
\begin{aligned}
R(\tau) & \approx \int_{\mathbb{R}^{3}} \frac{2 k_{B} T}{\rho L^{3}} \exp \left(-A|\mathbf{k}|^{2} \tau\right) d \mathbf{k} \\
& =\frac{8 \pi k_{B} T}{\rho L^{3}} \int_{0}^{\infty} k^{2} \exp \left(-A k^{2} \tau\right) d k \\
& =\frac{8 \pi k_{B} T}{\rho L^{3}} \frac{1}{2}\left(\sqrt{2 \pi \frac{1}{2 A \tau}} \frac{1}{2 A \tau}\right),
\end{aligned}
$$

where the second equality follows readily by using standard facts about Gaussians. In particular, the integral can be treated as the expectation of the second moment by introducing the standard normalization factor. Using [5.15 and simplifying the expression yields 5.12 ,

5.3. Equilibrium Statistics of Immersed Particles. For the particle-fluid system with the fixed temperature $T$, volume $V$, and number of elementary particles $M$, with the particles subject to a conservative force field, we have from statistical mechanics that the equilibrium probability density $\Psi$ of the elementary particle positions should have Boltzmann statistics:

$$
\Psi(\{\mathbf{X}\})=\frac{1}{Z} \exp \left(-\frac{\mathcal{E}(\{\mathbf{X}\})}{k_{B} T}\right),
$$

where $\mathcal{E}$ is the energy of a configuration of elementary particles. The factor $Z$ is the normalization factor so that the density integrates to one. The Boltzmann distribution arises from the thermodynamic condition that the equilibrium probability distribution 


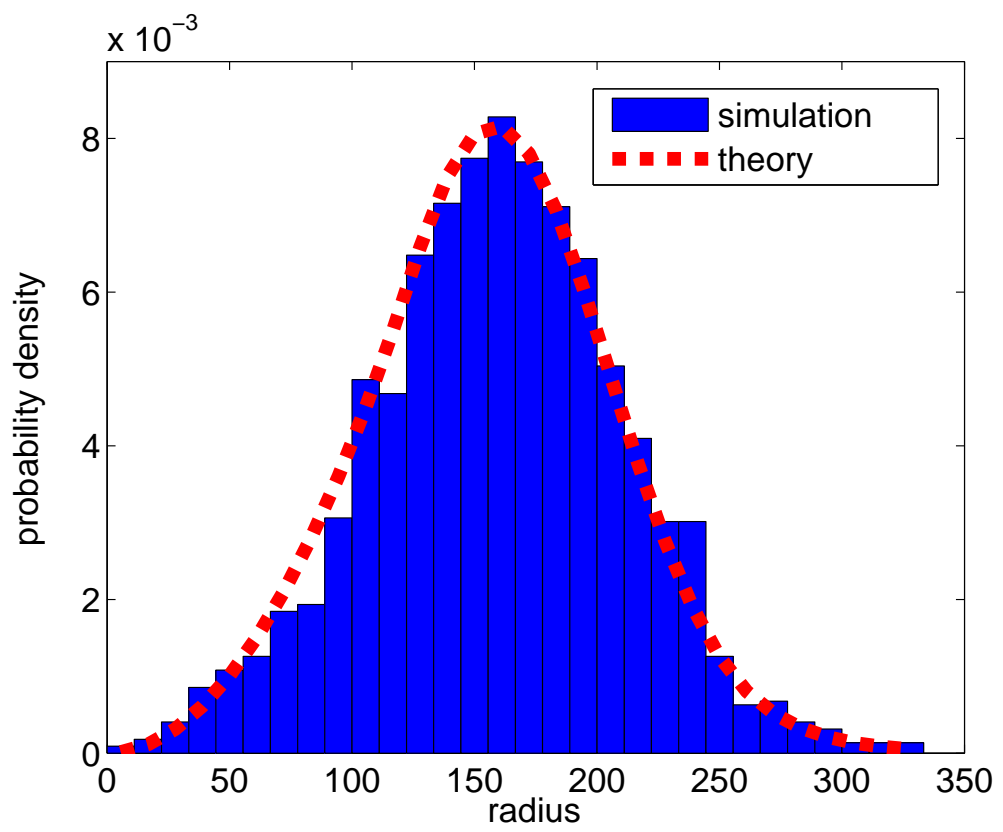

FIG. 5.2. Boltzmann Distribution vs Numerical Equilibrium Distribution. The spherical potential energy has the parameters $R_{1}=125, R_{2}=250, c=6 k_{B} T /\left(R_{2}-R_{1}\right)$. The simulation was run with the parameters $N=16, L=1000 \mathrm{~nm}, \Delta x=L / N, \mu=6.02 \times 10^{5} \mathrm{amu} /(\mathrm{nm} \cdot \mathrm{ns})$, $\rho=602 \mathrm{amu} / \mathrm{nm}^{3}, \mathrm{~T}=300 \mathrm{~K}, \Delta t=1000 \mathrm{~ns} .200,000$ time steps were simulated.

of the microscopic states maximize entropy while maintaining a fixed average energy for the system (70).

In deriving the thermal forcing of the system in Subsection 3.3.3. only the energy associated with the fluid modes was considered. When immersed structures are subject to a force, it is not entirely clear that the correct equilibrium distribution for the system as a whole will be attained.

When formulated in continuous time, the fluid-particle coupling in the immersed boundary method conserves energy exactly (64). While this may suggest that the correct equilibrium statistics should be obtained (up to the appropriate definition of an effective temperature), the discretization of time in the numerical method could in principle disrupt it, particularly since we are not employing a symplectic method (57; 74).

We now show that the numerical method from Section 3.1 appears to yield results consistent with the Boltzmann distribution, at least for the statistics of the position of a single immersed particle. For a more rigorous approach in which the Fokker-Plank equations associated with the stochastic immersed boundary method are analyzed, see the related work (3) .

To facilitate calculation of the equilibrium statistics, the particles are subject to 
a radially symmetric external force depending on $r=|\mathbf{X}|$ with the potential energy

$$
V(r)= \begin{cases}0, & r<R_{1} \\ c \cdot\left(r-R_{1}\right), & R_{1} \leq r \leq R_{2} \\ c \cdot\left(R_{2}-R_{1}\right), & r>R_{2} .\end{cases}
$$

It is assumed that $c>0$ so that this can be thought of physically as the potential associated with confining particles to a spherical chamber of radius $R_{2}$. The inner radius $R_{1}$ is used to soften the particle-wall interactions to avoid issues of numerical stiffness and $R_{2}$ is taken significantly smaller than the spatial period of the lattice $L$.

For a single immersed particle, the Boltzmann distribution for its radial coordinate is

$$
\tilde{\Psi}(r)=\frac{4 \pi r^{2}}{\tilde{Z}} \exp \left(-\frac{V(r)}{k_{B} T}\right)
$$

where $\tilde{Z}$ is the normalization factor.

In Figure 5.2, the equilibrium statistics of immersed particles simulated with the numerical method are compared with the Boltzmann distribution 5.19, The simulations were performed with $R_{1}=125 \mathrm{~nm}, R_{2}=250 \mathrm{~nm}$ and $c=6 k_{B} T /\left(R_{2}-R_{1}\right)$ with the parameters of the fluid-particle system given in Table 4.2 .

5.4. Osmotic Pressure of Confined Non-interacting Particles. Osmosis is a phenomenon that occurs in many microscale biological systems. When diffusing particles are confined to a chamber by a boundary which is permeable to fluid but less permeable to particles, a pressure difference develops between the inside and the outside of the chamber. This difference is referred to as the "osmotic pressure".

When the confining boundary is impermeable to particles and the system is in equilibrium, van't Hoff's law (70) relates the osmotic pressure to the concentration of the confined particles as

$$
p_{\text {Osmosis }}=\bar{c}_{0} k_{B} T \text {, }
$$

where $\bar{c}_{0}$ is the number of particles per unit volume in the chamber. More precisely, when the number of confined particles is small enough that the instantaneous pressure fluctuates, then van't Hoff's law should describe the ensemble or time average of the pressure difference that arises from confinement.

One should see a signature of van't Hoff's law in the fluid pressure when a collection of $M$ non-interacting particles in a conservative force field with potential $V$ are simulated by the stochastic immersed boundary method, given that the method was shown in Subection 5.3 to produce correct Boltzmann equilibrium statistics. Indeed, taking the expectation of the velocity, force, and pressure with respect to Boltzmann's distribution (ensemble average) in the fluid equation 2.1 gives

$$
0=-\nabla\langle p(\mathbf{x})\rangle+\left\langle\mathbf{f}_{\mathrm{prt}}(\mathbf{x})\right\rangle .
$$

This is obtained using that $\langle\mathbf{u}\rangle=0$ and $\left\langle\mathbf{f}_{\text {thm }}\right\rangle=0$. The notation $\langle\cdot\rangle$ denotes the ensemble average over the thermal fluctuations and $\langle p\rangle$ denotes the average of the fluid pressure field.

The ensemble average of the conservative force field with potential $V$ at location $\mathrm{x}$ is

$$
\left\langle\mathbf{f}_{\mathrm{prt}}(\mathbf{x})\right\rangle=\frac{-M \nabla V(\mathbf{x})}{Z} e^{-\frac{V(\mathbf{x})}{k_{B} T}}
$$




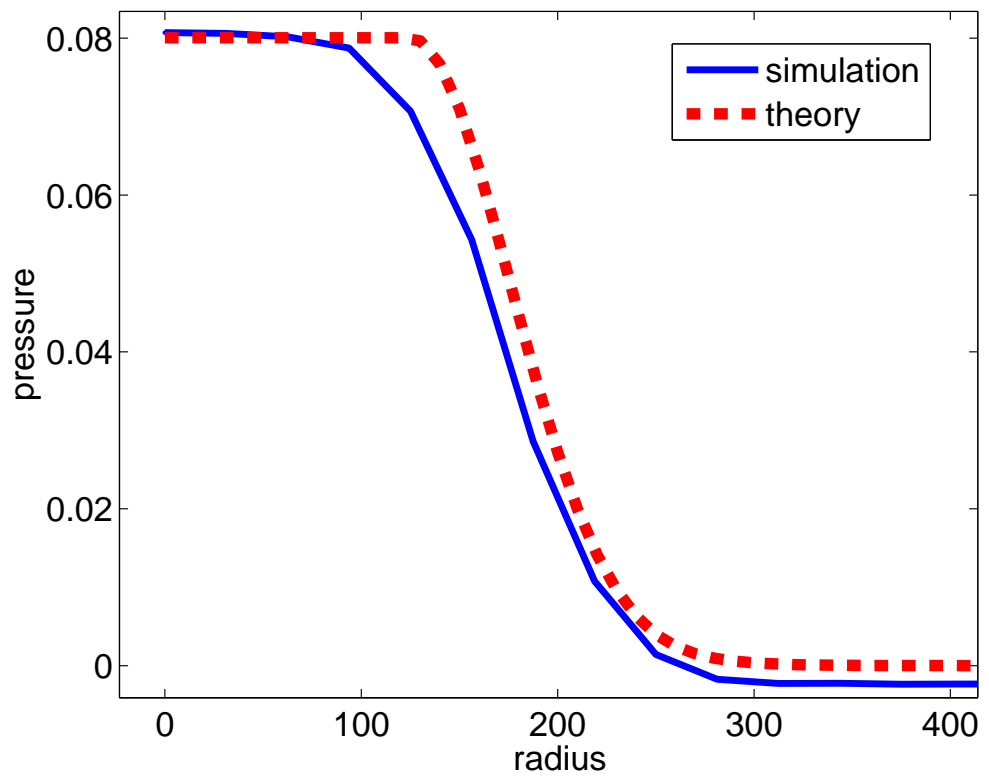

FIG. 5.3. Time Averaged Radial Pressure. The spherical potential energy has the parameters $R_{1}=125, R_{2}=250, c=6 k_{B} T /\left(R_{2}-R_{1}\right)$. The simulation was run with the parameters $N=16$, $L=1000 \mathrm{~nm}, \Delta x=L / N, \mu=6.02 \times 10^{5} \mathrm{amu} /(\mathrm{nm} \cdot \mathrm{ns}), \rho=602 \mathrm{amu} / \mathrm{nm}^{3}, \mathrm{~T}=300 \mathrm{~K}$, $\Delta t=1000$ ns. 200,000 time steps were simulated.

The average pressure $\langle p(\mathbf{x})\rangle$ can be determined up to an additive constant from 5.21 and 5.22 by taking the line integral in $\mathbf{x}$. From the fundamental theorem of line integrals with the additive constant set to zero (to give the appropriate decay at large $\mathbf{x})$ we have

$$
\langle p(\mathbf{x})\rangle=\frac{M}{Z} e^{-\frac{V(\mathbf{x})}{k_{B} T}} k_{B} T
$$

By the assumption of Boltzmann statistics for the immersed particles the concentration field is

$$
c_{0}(\mathbf{x})=\frac{M}{Z} e^{-\frac{V(\mathbf{x})}{k_{B} T}} .
$$

Substitution into 5.23 and integrating over the chamber gives van't Hoff's law. This derivation also indicates that the stochastic immersed boundary method has a fluid pressure field which should respect the local formulation of van't Hoff's law:

$$
p_{0}(\mathbf{x})=c_{0}(\mathbf{x}) k_{B} T .
$$

From a statistical mechanical point of view of this derivation, this fluid pressure can be thought of as arising from the fluctuations of the system which persistently 
subject a particle to the confining forces in the vicinity of the wall with a frequency determined by the Boltzmann statistics. The forces are then transferred to the fluid by the viscous particle-fluid interactions. Similar "mesoscopic" points of view of osmosis have been used in $(4 ; 21 ; 61)$.

In Figure 5.3. a comparison is made of the pressure predicted by a local van't Hoff's law taking into account the local solute concentration and the time average of the pressure field of the fluid obtained in a numerical simulation using the stochastic immersed boundary method for an immersed particle confined by the spherically symmetric potential given by equation 5.18 .

Another quantification of osmotic pressure is the average force per unit area exerted on the walls of the chamber which confine the solute. For a spherical chamber $\Omega$ of radius $R$ in which the solute exerts a (generally repulsive) areal force density $\mathbf{F}_{\mathrm{pw}}(\mathbf{z})$ on a portion of the wall boundary $\partial \Omega$ at relative location $\mathbf{z}$, this osmotic pressure is given by:

$$
p_{\text {wall }}=\frac{1}{4 \pi R^{2}} \int_{\Omega} \int_{\partial \Omega} \mathbf{F}_{\mathrm{pw}}(\mathbf{y}-\mathbf{x}) \cdot \frac{\mathbf{y}}{|\mathbf{y}|} c(\mathbf{x}) d \mathbf{y} d \mathbf{x}
$$

where $c(\mathbf{x})$ denotes the average concentration of the solute particles. Note that this formula applies also when the solute particles interact with each other. We will only be considering isotropic wall-solute interactions (and uniform distribution of wall molecules) so that we can write the force density in terms of a (typically nonnegative) scalar function $q_{\mathrm{pw}}(r): \mathbf{F}_{\mathrm{pw}}(\mathbf{z})=q_{\mathrm{pw}}(|\mathbf{z}|) \mathbf{z} /|\mathbf{z}|$ and the concentration density as $c(\mathbf{x})=$ $c(|\mathbf{x}|)$. The expression in equation 5.26 for the osmotic pressure on the chamber wall can then be simplified by integrating over the angular degrees of freedom:

$$
p_{\text {wall }}=\frac{1}{R^{2}} \int_{0}^{R} h(r) c(r) r^{2} d r
$$

with

$$
\begin{aligned}
h(|\mathbf{x}|) & =\int_{\partial \Omega} \mathbf{F}_{\mathrm{pw}}(|\mathbf{y}-\mathbf{x}|) \cdot \frac{\mathbf{y}}{|\mathbf{y}|} d \mathbf{y} \\
& =\frac{\pi}{r} \int_{R-r}^{R+r} q_{\mathrm{pw}}(\rho)\left(\rho^{2}+R^{2}-r^{2}\right) d \rho .
\end{aligned}
$$

The function $h(r)$ can be interpreted as the integrated normal force applied to the wall by a solute particle located a distance $r=|\mathbf{x}|$ from the origin. The change of variable used to obtain the last integral was $\rho=|\mathbf{y}-\mathbf{x}|$.

When the potential confining the solute is "hard-walled," in the sense that the solute-wall interactions occur only in a very small boundary layer of the wall, the two formulas 5.27 and 5.23 give the same values for the osmotic pressure, up to a small difference which vanishes as the width of the boundary layer is taken to zero. For potentials which are "soft-walled" in the sense that solute molecules interact with the wall on a length scale comparable to the magnitude of the fluctuations of the size of the solute molecules, as in Subsection 5.5, the average pressure of the fluid and the average pressure exerted on the wall may in fact differ. In related work, we are investigating the various pressures associated with osmotic phenomena and exploring the influence of finite wall and molecule sizes (5). For a discussion of how the osmotic pressure can be used to drive fluid flow in a mesoscopic pump, see the related work (4). We now present a few examples to demonstrate how more complex structures immersed 
in the fluid can be simulated with the stochastic immersed boundary method and to show how the osmotic pressure associated with wall forces can be derived from the thermal fluctuations of these structures as simulated by the method.

Before proceeding in the following subsections to consider osmotic pressure effects of more complex structures, we remark that the wall pressure can be computed from the average of the radial confinement force $\mathbf{~}_{\text {conf }}(\mathbf{x})=-f_{\text {conf }}(|\mathbf{x}|) \mathbf{x} /|\mathbf{x}|$ acting on the particles and equation 5.26 in the case of a spherical chamber. From Newton's third law (principle of equal and opposite forces):

$$
\mathbf{f}_{\mathrm{conf}}(\mathbf{x})=\int_{\partial \Omega}-\mathbf{F}_{\mathrm{pw}}(\mathbf{y}-\mathbf{x}) d \mathbf{y},
$$

re-expressed using the isotropy of the forces involved, we have:

$$
-f_{\text {conf }}(|\mathbf{x}|) \frac{\mathbf{x}}{|\mathbf{x}|}=\int_{\partial \Omega}-q(|\mathbf{y}-\mathbf{x}|) \frac{\mathbf{y}-\mathbf{x}}{|\mathbf{y}-\mathbf{x}|} d \mathbf{y} .
$$

In spherical coordinates, this can be written:

$$
f_{\text {conf }}(r)=\frac{\pi R}{r^{2}} \int_{R-r}^{R+r} q(\rho)\left(r^{2}-R^{2}+\rho^{2}\right) d \rho .
$$

If the model for the solute-wall interaction force can be assumed to vanish at separation distances comparable to the chamber radius, then this integral relation between $q$ and $f$ can be inverted to obtain:

$q(\rho)=\left(\frac{1}{2 \pi R \rho^{2}}\right)\left(\rho(R-\rho) f_{\text {conf }}^{\prime}(R-\rho)+(R+\rho) f_{\text {conf }}(R-\rho)+\int_{0}^{R-\rho} f_{\text {conf }}(s) d s\right)$.

The pressure on the wall can then be computed from the effective bulk confinement force $f_{\text {conf }}(r)$ using 5.27, 5.28, and this inversion formula.

5.5. Application: Simulation of Interacting Immersed Particles and Osmotic Pressure. We now discuss application of the stochastic immersed boundary method in determining the osmotic pressure when the confined particles can interact. In particular, we consider the case in which particles interact in distinct pairs (dimers) through a spring with non-zero rest length and are confined to an approximately $400 \mathrm{~nm}$ spherical chamber. Note that the solute particles are confined in a microscopic chamber, in the sense that the chamber diameter is comparable or smaller than the length-scale associated with the solute particle interactions between the monomers. This is in contrast to a macroscopic chamber in which solute particles interact on a length-scale very much smaller than the chamber diameter and where the van't Hoff law is well established with the osmotic pressure depending only on the number of solute particles and not on their physical characteristics. For example, in microscopic chambers the amplitude of the fluctuations of a solute particle's diameter may be comparable to the chamber diameter and play a non-negligible role in the osmotic pressure associated with confinement.

To investigate these effects, we consider how the osmotic pressure changes as the binding strength for a collection of dimers is varied. From the classical van't Hoff's law, it would be expected that the osmotic pressure for tightly bound dimers are half 
that of the zero-binding case (free monomers), because a tightly bound dimer behaves effectively as a single particle. This concept is exploited to suggest the design of an osmotically driven pumping apparatus in (4). From simulations using the stochastic immersed boundary method, we can investigate how the osmotic pressure varies between the unbound and tightly bound regimes.

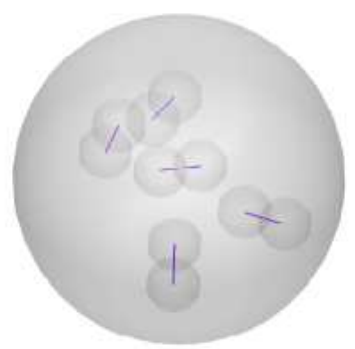

FIG. 5.4. Illustration of five distinct pairs of coupled particles confined in a spherical chamber with the soft-wall potential given by equation 5.34. Simulations were performed for five pairs of particles coupled with interaction energy given by equation 5.33

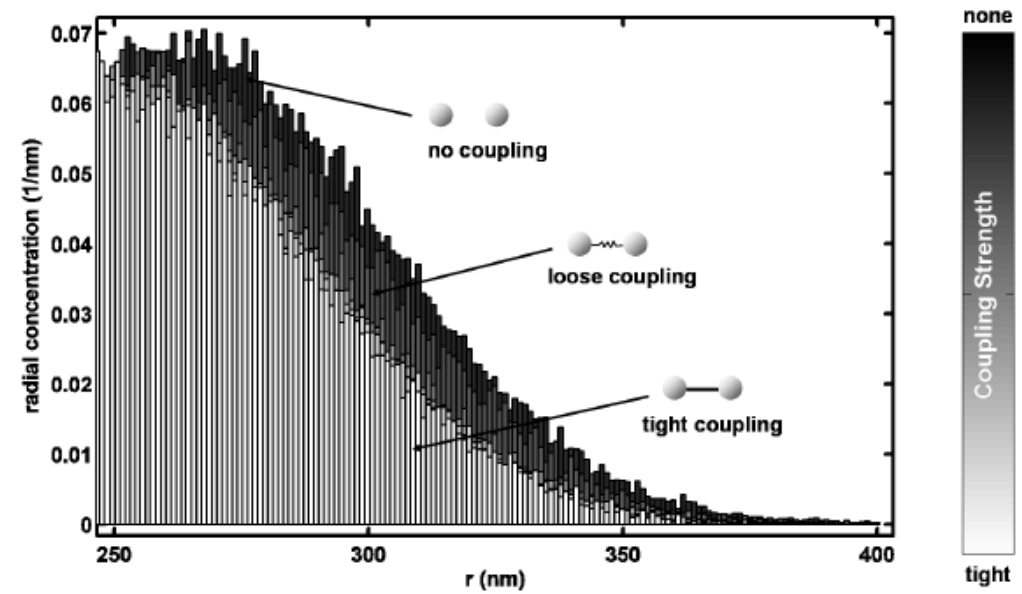

FIG. 5.5. The geometrically weighted particle concentration $c(|\mathbf{x}|=r) r^{2}$ of the monomers in the spherical shell of radius $r$ used in equation 5.27. As the coupling is tightened, the particle concentration has more weight at smaller radii. The concentration is shown only over the boundary layer over which the confinement force is exerted.

Each pair of particles is coupled by a potential energy corresponding to a standard spring model with finite rest length $\ell$ :

$$
\Phi_{2}\left(\mathbf{X}_{1}, \mathbf{X}_{2}\right)=\frac{K}{2}\left(\left|\mathbf{X}_{1}-\mathbf{X}_{2}\right|-\ell\right)^{2},
$$

where $\mathbf{X}_{1}$ and $\mathbf{X}_{2}$ denote the particle locations and $K$ represents the spring stiffness. Each monomer at location $\mathbf{x}$ is subject to a confinement force given by the radially 
symmetric potential:

$$
\Phi_{1}(\mathbf{x})= \begin{cases}0, & |\mathbf{x}| \leq R_{1}, \\ \frac{C_{0}}{2}\left(|\mathbf{x}|-R_{1}\right)^{2}, & R_{1} \leq|\mathbf{x}| \leq R_{2} \\ \frac{C_{0}}{2}\left(R_{2}-R_{1}\right)^{2}, & |\mathbf{x}| \geq R_{2} .\end{cases}
$$

This potential can be thought of as arising from the interaction force of the confined solute monomers with particles distributed uniformly over the walls of a spherical chamber having radius $R=R_{2}$. The formula given in equation 5.32 gives the relationship between the monomer-wall interaction force and the radial confinement force. For the simulations the parameters were chosen as $\ell=100 \mathrm{~nm}, R_{1}=375 \mathrm{~nm}$, $R_{2}=400 \mathrm{~nm}, C_{0}=8 k_{B} T /\left(R_{2}-R_{1}\right)^{2}$. The setup is depicted pictorially in Figure 5.4 and movies of the simulations can be found in the Supplemental Materials.

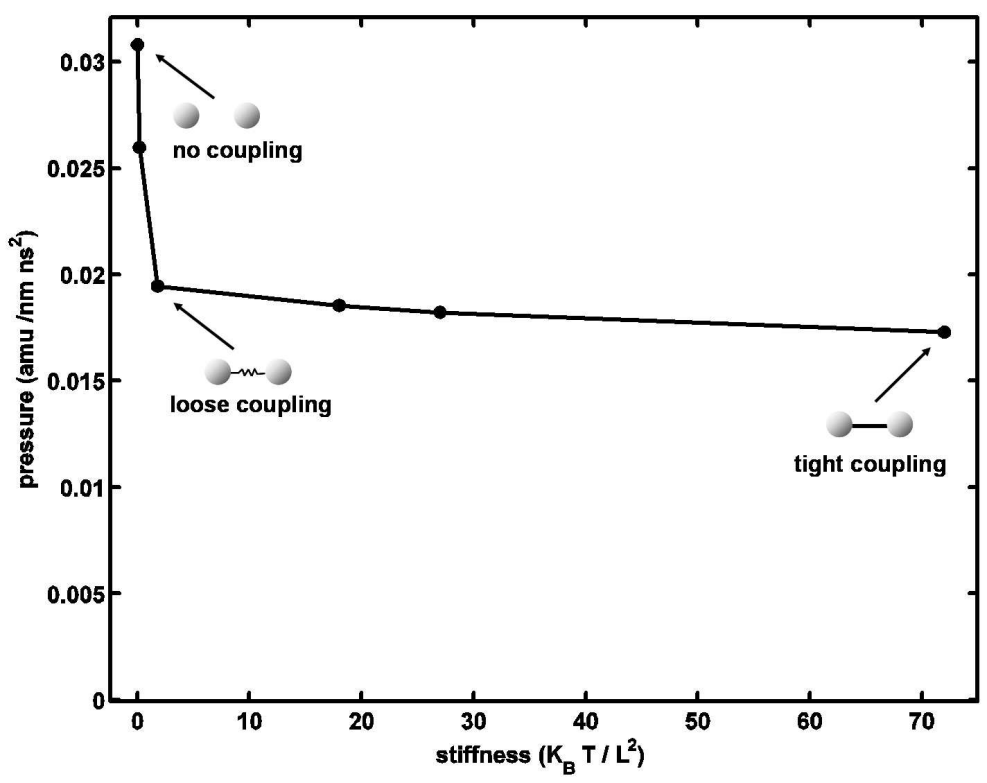

FIG. 5.6. The osmotic pressure for confined dimers as a function of coupling strength. As the coupling strength of the dimers increases, the osmotic pressure decreases in a non-linear manner. The osmotic pressure value for unbound monomers is approximately double that of the the tightly bound monomers, which is in accordance with what is expected under van't Hoff's law. Deviations from van't Hoff's law are apparent for intermediate coupling strengths for which the length scale of the dimers is comparable to those of the chamber and the wall thickness.

Figure 5.5 displays the results of simulations with the stochastic immersed boundary method that show that as the stiffness is increased, the particle density decreases for each radius $r$ within the region of the confining potential. The pressure consequently drops with increasing coupling stiffness, as shown in Figure 5.6. We see also that, in accordance with van't Hoff's law, the pressure in the strong coupling limit, where the particle pairs behave effectively as single entities, is cut to roughtly half from the no coupling case. We observe deviations from the classical van't Hoff law when the length scale of the bound molecules is comparable to that of the wall or the chamber.

An intuitive statistical explanation for the pressure drop is that a strongly coupled particle is less likely to venture far into the confining potential because roughly 
half the time its partner will encounter the confining potential first, be repelled, and pull its accompanying particle back away from the confining potential sooner than it would have on its own. In more physical terms, the entropy of the particle pairs decreases and consequently the entropic penalty associated with confinement is reduced as the coupling strength increases. For coupling values that make the dimer length scale comparable to the microscopic chamber size, the osmotic pressure assumes an intermediate value which is not well described by a van't Hoff's law.

5.6. Application: Simulation of Polymer Chains and Polymer Knots. A fundamental feature of the stochastic immersed boundary method is that each structure evolves according to a local average of a common fluid velocity field. The method therefore automatically captures the physical phenomenon that the velocities of immersed structures become strongly correlated when they are close together in space. Mathematically, the solution map of the immersed structures and surrounding fluid volume, which maps a configuration of the fluid and structures at a reference time to the solution configuration at a later time $t$, can be viewed as a homeomorphism. Consequently, in the continuous-time framework, the method preserves topological invariants of the immersed structures, such as the knottedness of a continuous closed curve, as they evolve. This is in contrast to other simulation methods, such as Stokesian Dynamics (15; 75; 77), which would require explicit excluded volume constraints and/or repulsion forces between monomers to prevent topological changes.

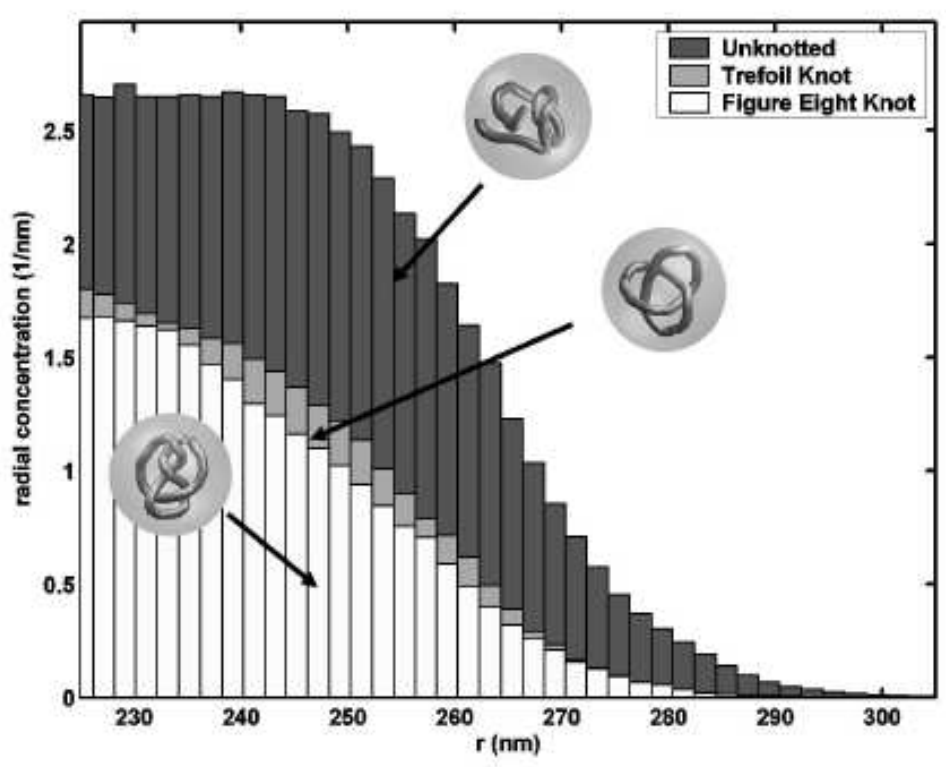

FIG. 5.7. The bar graph shows the average concentration of monomers within the chamber in the boundary layer in which they interact with the confinement forces (Subsection 5.4). As the knottedness of the polymer increases, the monomers are more restricted and concentrate on average toward the chamber center spending less time interacting with the confinement forces. As a consequence the concentration of monomers in the boundary layer decreases and the overall pressure drops (Table 5.1). The parameters in the simulations were taken the same as in Subsection 5.4

To demonstrate this feature of the method in practice (with finite time step) and 
to show how worm-like chain polymers can be simulated, the stochastic immersed boundary method was applied for a generic polymer chain, a polymer trefoil knot, and a polymer figure eight knot. From these simulations the osmotic pressure of confinement was estimated for each of the polymers. The results of the average concentration of the polymer monomers, which determine the average radial force density exerted on the confining wall, is given in Figure 5.7

As the knottedness of the polymer increases, its constituent monomers spend less time at large radii, and as seen in Table [5.1, the osmotic pressure is significantly reduced. An intuitive explanation is that as the knottedness of the polymer increases, this restricts the intrinsic configurations accessible to the thermally fluctuating polymer. In physical terms, the knottedness reduces the entropic penalty of confining of the polymer. Movies showing simulations of the thermally fluctuating polymer knots can be found in the Supplemental Materials.

TABLE 5.1

Osmotic Pressure of Polymer Knots

\begin{tabular}{|l|l|}
\hline Knot Type & Osmotic Pressure $\left(\mathrm{amu} / \mathrm{nm} \cdot \mathrm{ns}^{2}\right)$ \\
\hline Unknotted & 0.16 \\
Trefoil Knot & 0.0439 \\
Figure Eight Knot & 0.0392 \\
\hline
\end{tabular}

5.7. Application: Simulation of a Basic Model for a Molecular Motor Protein Transporting a Membrane-Bound Cargo Vesicle . We now discuss how more complex systems can be simulated with the immersed boundary method. On a subcellular level motor proteins interact with cytoskeletal structures, such as actin and microtubules, to generate force and to transport materials within the cell. For example, neurotransmitters are produced in the cell body of neurons and transported by kinesin motor proteins along axons to the vicinity of the synaptic cleft where they are packaged for future release (8). We demonstrate how the stochastic immersed boundary method can be applied to simulate a basic model of a molecular motor protein immersed in a fluid moving along a filament which transports a cargo vesicle (Figure 5.8).

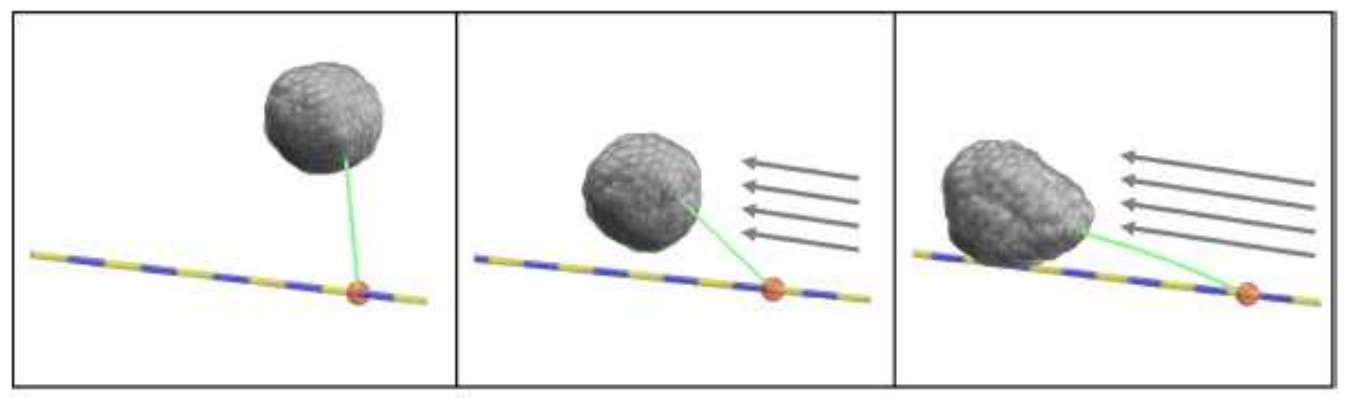

FIG. 5.8. Illustration of the basic model of a molecular motor protein immersed in a fluid and towing a cargo vesicle. As the fluid flow strengthens, the hydrodynamic drag on the cargo increases and a load force is exerted in opposition to the motor transport. For large opposing fluid flows, the cargo may significantly change shape in response to the flow. 
The motor protein is modeled as a Brownian Ratchet $(41 ; 66)$ and the cargo vesicle is modeled by a triangulated mesh which forms a membrane enclosing a spherical volume. The nodes of the mesh are linked together by springs of the form given in equation 5.33 with non-zero rest lengths determined by the distance between nodes in an initial spherical configuration. The cargo is linked at the vesicle surface to the motor by a spring of the form given by equation 5.33 with a non-zero rest length of approximately $100 \mathrm{~nm}$. The spherical vesicle has a radius of $125 \mathrm{~nm}$ and the ratcheting intervals (light and dark inset of Figure [5.9) are of length $100 \mathrm{~nm}$.

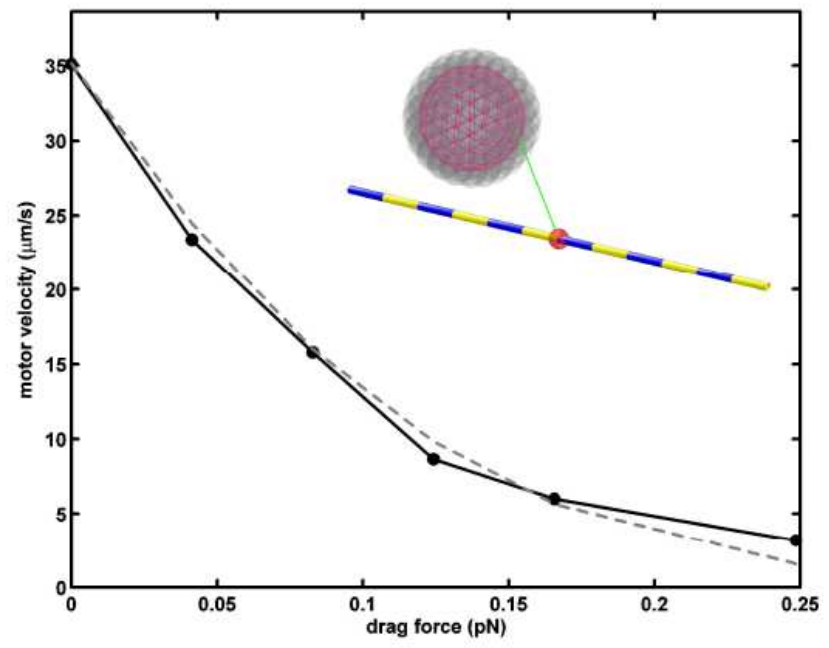

FIG. 5.9. The mean motor velocity vs the hydrodynamic drag force. The data points joined by the solid curve shows the mean motor velocity $X_{\tau} / \tau$ obtained from simulations over approximately $\tau=3 \mathrm{~ms}$. The dashed curve shows the mean velocity for an idealized ratchet having a viscous drag comparable to the spherical vesicle and subject to a constant load force the same strength as the hydrodynamic drag force $(41 ;$;66).

To examine how the mean velocity of transport by the motor protein behaves under different loading conditions, simulations were performed in which a hydrodynamic drag force is generated on the vesicle cargo by a bulk flow of the fluid $(\mathbf{8} ; 41)$. The mean velocity for different strengths of the countering fluid flow is plotted in Figure 5.9, where we see that for significantly large opposing flow, the motor can almost be made to "stall". The stochastic immersed boundary method allows for hydrodynamic effects associated with the shape and deformation of cargos to be investigated. These effects, not typically considered in other numerical simulation approaches for molecular motors, may have important consequences for motor/polymerization ratchet transport when more details of the motor are taken into account or with more complex cargos such as membrane tubes, small cell organelles, or chromosomes $(8 ;$; $33 ; 69)$. More sophisticated models can also be formulated within the stochastic immersed boundary method framework, such as the case in which multiple motor proteins transport a common cargo, interact by crosslink cytoskeletal filaments, or include additional mechanical degrees of freedom of the motor protein itself. Some movies of our motor protein model transport simulations can be found in the Supplemental Materials. 
6. Conclusion and Discussion. In this work we have discussed how thermal fluctuations can be incorporated into the immersed boundary method in a manner consistent with the laws of statistical mechanics. A new stochastic numerical method was proposed that allows for a range of time steps to be taken in which the fastest degrees of freedom of the fluid-particle system are either underresolved, partially resolved, or fully resolved. In addition, the numerical method was designed to take into account in a systematic way the statistical contributions of the thermal fluctuations over long time steps with the correct correlations between the particles and fluid.

To investigate the behavior of the immersed boundary framework and the stochastic numerical method with respect to well-known laws in statistical physics a number of theoretical results were obtained for the method and compared with numerical simulations. In particular, it was shown that immersed particles simulated with the numerical method exhibit the correct scaling in the physical parameters for the mean squared displacement in three dimensions. It was also shown that the stochastic numerical method captured inertial effects of the fluid with a velocity autocorrelation function for a particle that for long times decays with algebraic order $\tau^{-3 / 2}$. We further found that particles appear to have the correct Boltzmann equilibrium statistics. Moreover, the method was found to produce the van't Hoff law of osmosis for a particle confined to a spherical chamber recovering the correct osmotic pressure. In addition results were presented which showed how the osmotic pressure could be computed for interacting pairs of particles and worm-like chain polymers, including a trefoil and figure eight polymer knot. A more complex application of a basic model of a molecular motor protein immersed in a fluid and towing a vesicle-bound cargo subject to a hydrodynamic drag force was simulated and the force-velocity statistics computed.

These basic physical checks indicate that the stochastic immersed boundary method has the capability to capture many important features of thermally fluctuating systems involving immersed structures which interact with a fluid. The results presented suggest the promise of the stochastic immersed boundary method as an effective approach in modeling and simulating the mechanics of biological systems at the cellular and intracellular level.

Acknowledgments. The author P.J.A was supported by NSF VIGRE Postdoctoral Research Fellowship Grant DMS - 9983646 and NSF Mathematical Biology Grant DMS - 0635535. The authors would like to thank Eric Vanden-Eijnden and David Cai for helpful discussions on analytical and physical aspects of the work, and Tom Bringley for a careful reading of a preliminary draft. The authors also thank David McQueen for discussions concerning the immersed boundary method and Yuri Lov for lending computational resources used to obtain some of the numerical results. We are especially indebted to George Oster, whose vision of direct numerical simulation of osmotic phenomena via random forces inspired this work.

Appendix A. The Representation Function $\delta_{a}$ for Immersed Particles. In the immersed boundary method, it is required that a function $\delta_{a}$ be specified to represent the elementary particles. The representation of this function is often derived from the following function $\phi$ which is known to have desirable numerical properties 
(64):

$$
\phi(r)= \begin{cases}0 & , \text { if } r \leq-2 \\ \frac{1}{8}\left(5+2 r-\sqrt{-7-12 r-4 r^{2}}\right) & , \text { if }-2 \leq r \leq-1 \\ \frac{1}{8}\left(3+2 r+\sqrt{1-4 r-4 r^{2}}\right) & , \text { if }-1 \leq r \leq 0 \\ \frac{1}{8}\left(3-2 r+\sqrt{1+4 r-4 r^{2}}\right) & , \text { if } 0 \leq r \leq 1 \\ \frac{1}{8}\left(5-2 r-\sqrt{-7+12 r-4 r^{2}}\right) & , \text { if } 1 \leq r \leq 2 \\ 0 & , \text { if } 2 \leq r .\end{cases}
$$

For three dimensional systems the function $\delta_{a}$ representing elementary particles of size $a$ is

$$
\delta_{a}(\mathbf{r})=\frac{1}{a^{3}} \phi\left(\frac{\mathbf{r}^{(1)}}{a}\right) \phi\left(\frac{\mathbf{r}^{(2)}}{a}\right) \phi\left(\frac{\mathbf{r}^{(3)}}{a}\right),
$$

where the superscript indicates the index of the vector component.

To maintain good numerical properties, the particles are restricted to sizes $a=$ $n \Delta x$, where $n$ is a positive integer. For a derivation and a detailed discussion of the properties of these functions see (64).

Appendix B. The Fourier Coefficients of the Function $\delta_{a}$ Used to Represent an Immersed Particle. Throughout the paper it will be useful to consider the Fourier coefficients of the function $\delta_{a}(\mathbf{x}-\mathbf{X})$ used to represent an elementary particle situated at position $\mathbf{X}$. While the function is defined for all $\mathbf{x} \in \Lambda$, it is often useful to consider the restriction of the function to the discrete lattice points $\left\{\mathbf{x}_{\mathbf{m}}=\mathbf{m} \Delta x \mid \mathbf{m} \in \mathbb{Z}_{N}^{3}\right\}$.

We will use the following notation to denote the discrete Fourier transform of the delta function restricted to the lattice:

$$
\hat{\delta}_{a, \mathbf{k}}(\mathbf{X})=\frac{1}{N^{3}} \sum_{\mathbf{m}} \delta_{a}\left(\mathbf{x}_{\mathbf{m}}-\mathbf{X}\right) \exp (-i 2 \pi \mathbf{k} \cdot \mathbf{m} / N) .
$$

The dependence of the Fourier coefficients on the particle position $\mathbf{X}$ (relative to the lattice) is explicitly noted. When the dependence on $\mathbf{X}$ is not explicitly noted, then we will be referring implicitly to the discrete Fourier transform of the delta function when centered on a lattice point: $\hat{\delta}_{a, \mathbf{k}}:=\hat{\delta}_{a, \mathbf{k}}(\mathbf{0})$.

Appendix C. Autocorrelation Function for the Velocity Field of the Fluid. In this section the autocorrelation function is computed for the velocity field of the fluid in the absence of force $\mathbf{f}_{\mathrm{prt}}=0$. This is done by representing the velocity field in Fourier space and computing the autocorrelation function of each mode $\mathbf{k}$. From equation 3.56 and standard stochastic calculus the steady-state autocorrelation function of the $\mathbf{k}^{t h}$ mode when $s>r$ is

$$
=2 D_{\mathbf{k}} E\left(\overline{\int_{-\infty}^{s} e^{-\alpha_{\mathbf{k}}(r-w)} \wp_{\mathbf{k}}^{\perp} d \tilde{\mathbf{B}}_{\mathbf{k}}(w)} \cdot \int_{-\infty}^{r} e^{-\alpha_{\mathbf{k}}(s-q)} \wp_{\mathbf{k}}^{\perp} d \tilde{\mathbf{B}}_{\mathbf{k}}(q)\right),
$$


where the notation $\wp_{\mathbf{k}}^{\perp}$ denotes projection orthogonal to $\hat{\mathbf{g}}_{\mathbf{k}}$ as defined in Subsection 3.3 .2 .

By applying Ito's Isometry to C.2, and observing the symmetry under the interchange $s \leftrightarrow r$, the autocorrelation function is given by

$$
\begin{aligned}
E\left(\overline{\hat{\mathbf{u}}_{\mathbf{k}}(s)} \cdot \hat{\mathbf{u}}_{\mathbf{k}}(r)\right) & = \begin{cases}3 \frac{D_{\mathbf{k}}}{\alpha_{\mathbf{k}}} e^{-\alpha_{\mathbf{k}}|s-r|} & \text { if } \mathbf{k} \in \mathcal{K} \\
4 \frac{D_{\mathbf{k}}}{\alpha_{\mathbf{k}}} e^{-\alpha_{\mathbf{k}}|s-r|} & \text { if } \mathbf{k} \notin \mathcal{K}\end{cases} \\
& =\Upsilon_{\mathbf{k}} \frac{\frac{k}{B}_{B} T}{\rho L^{3}} e^{-\alpha_{\mathbf{k}}|s-r|},
\end{aligned}
$$

where

$$
\Upsilon_{\mathbf{k}}= \begin{cases}3, & \mathbf{k} \in \mathcal{K} \\ 2, & \mathbf{k} \notin \mathcal{K}\end{cases}
$$

and the index set $\mathcal{K}$ is defined in 3.29

The factor $\Upsilon_{\mathbf{k}}$ arises from the incompressibility constraint 3.22, the real-valuedness constraint 3.25, and the dimensionality of the space orthogonal to $\hat{\mathbf{g}}_{\mathbf{k}}$. See Subsection 3.3 .3 for a discussion of how the constraints affect $D_{\mathbf{k}}$.

The spatio-temporal correlation function of the velocity field $\mathbf{u}$ is then given by

$$
\begin{aligned}
E\left(\mathbf{u}_{\mathbf{m}}(s) \cdot \mathbf{u}_{\mathbf{n}}(r)\right) & =\sum_{\mathbf{k}} \sum_{\mathbf{k}^{\prime}} E\left(\overline{\hat{\mathbf{u}}_{\mathbf{k}}(s)} \cdot \mathbf{u}_{\mathbf{k}^{\prime}}(r)\right) \exp \left(i 2 \pi\left(\mathbf{n} \cdot \mathbf{k}^{\prime}-\mathbf{m} \cdot \mathbf{k}\right) / N\right) \\
& =\sum_{\mathbf{k}} E\left(\overline{\hat{\mathbf{u}}_{\mathbf{k}}(s)} \cdot \hat{\mathbf{u}}_{\mathbf{k}}(r)\right) \exp (i 2 \pi(\mathbf{n}-\mathbf{m}) \cdot \mathbf{k} / N) \\
& =\frac{k_{B} T}{\rho L^{3}} \sum_{\mathbf{k}} \Upsilon_{\mathbf{k}} e^{-a_{\mathbf{k}}|s-r|} \exp (i 2 \pi(\mathbf{n}-\mathbf{m}) \cdot \mathbf{k} / N) .
\end{aligned}
$$

To obtain the second equality, we used the statistical independence of the Fourier modes of the velocity field when the indices $\mathbf{k}$ and $\mathbf{k}^{\prime}$ are distinct and do not correspond to conjugate modes (see 3.25). When the indices $\mathbf{k}$ and $\mathbf{k}^{\prime}$ do refer to conjugate but distinct modes, then the average vanishes because a mean zero random variable $Z$ with independent and identically distributed real and imaginary components satisfies $\left\langle Z^{2}\right\rangle=0$. The last equality follows by substitution from equation C.3.

Appendix D. Constants: Accuracy and Error Estimates. The nondimensional factors $Q$ appearing in the error estimates in Section 4 are approximately independent of the physical parameters. For comparison of the theoretical error estimates with numerical simulations, it is useful to compute the factors for specific physical parameters to obtain estimated values. Evaluation of the expressions for the $Q$ constants for the system with parameters given in Table 4.2 gives the following values:

$$
\begin{aligned}
& Q_{1}=0.563 \\
& Q_{2}=7.87 .
\end{aligned}
$$




\section{References.}

[1] P. J. Atzberger and C.S Peskin, A Three Dimensional Brownian Dynamics Model of Kinesin Incorporating the Coiled-Coil Cargo Tether, Bulletin of Mathematical Biology, (to appear)

[2] P. J. Atzberger and P. R. Kramer, Error Analysis of a Stochastic Immersed Boundary Method Incorporating Thermal Fluctuations, (submitted 2006)

[3] P. J. AtZBerger, Stochastic Analysis of the Immersed Boundary Method with Thermal Fluctuations, (in preparation 2006)

[4] P. J. Atzberger and Charles S. Peskin, Pumping Fluids with Osmotic Effects (in preparation 2006)

[5] P. J. Atzberger and Peter R. Kramer, A Microscopic Theory of Osmosis (in preparation 2006)

[6] B. J. Alder and T. E. Wainwright, Decay of the Velocity Autocorrelation Function, Phys. Rev. A, vol. 1 num. 1 (1970), pp. 18-21.

[7] R. J. AdLeR, On excursion sets, tube formulas and maxima of random fields, Annals of Applied Probability, 10 (2000), pp. 1-74.

[8] B. Alberts, A. Johnson, J. Lewis, M. Raff, K. Roberts, And P. Walker, Molecular Biology of the Cell, Garland Publishing, 2002.

[9] J. B. Avalos And A. D. Mackie, Dissipative particle dynamics with energy conservation, Europhys. Lett., 40 (1997), pp. 141-146.

[10] D. A. Beard AND T. Schlick, Inertial stochastic dynamics. II. Influence of inertia on slow kinetic processes of supercoiled DNA, J. Comp. Phys, 112 (2000), pp. 7323-7338.

[11] D. A. BeARD And T. Schlick, Computational modeling predicts the structure and dynamics of chromatin fiber, Structure, 9 (2001), pp. 105-114.

[12] D. BoAl, Mechanics of the Cell, Cambridge University Press, Cambridge, U.K., 2002.

[13] L. Bocquet, From a stochastic to a microscopic approach to Brownian motion, Acta Physica Polonica B, 29 (1998), pp. 1551-1564.

[14] G. Bossis and J. F. Brady, Dynamic simulation of sheared suspensions. I. General method, J. Chem. Phys., 80 (1984), pp. 5141-5154.

[15] J. F. Brady and G. Bossis, Stokesian dynamics, in Annual review of fluid mechanics, vol. 20 of Annu. Rev. Fluid Mech., Annual Reviews, Palo Alto, CA, 1988, pp. $111-157$.

[16] E. G. D. Cohen, Fundamental Problems in Statistical Mechanics III, International Summer School on Fundamental Problems in Statistical Mechanics, (1974).

[17] S. Corrsin, Atmospheric Diffusion and Air Pollution, edited by F. N. Frenkiel and P.A. Shappard, vol. 6, 1960.

[18] W. K. Den Otter and J. H. R. Clarke, A new algorithm for dissipative particle dynamics, Europhys. Lett., 53 (2001), pp. 426-431.

[19] J. M. Deutch And I. Oppenheim, The concept of Brownian motion in modern statistical mechanics, in Brownian Motion, vol. 83 of Faraday Discuss. Chem. Soc., London, 1987, The Faraday Division of the Royal Society of Chemistry, The Royal Society of Chemistry, pp. 1-20.

[20] J. R. Dorfman and E. G. D. Cohen, Velocity Correlation Functions in Two and Three Dimensions, Phys. Rev. Lett, vol. 25, num. 18, (1970), pp. 1257-1260.

[21] A. Einstein, Investigations on the Theory of the Brownian Movement, Dover Publishing, New York, (1956).

[22] D. L. Ermak and J. A. MCCammon, Brownian dynamics with hydrodynamic 
interactions, J. Chem. Phys., 69 (1978), pp. 1352-1360.

[23] M. H. Ernst and E. H. Hauge and J. M. J. van Leeuwen, Asymptotic Time Behavior of Correlation Functions.I. Kinetic Terms, Phys. Rev. A, vol. 4, num. 5, (1971), pp. 2055-2065.

[24] P. Español, Dissipative particle dynamics with energy conservation, Europhys. Lett., 40 (1997), pp. 631-636.

[25] P. Español and P. Warren, Statistical mechanics of dissipative particle dynamics, Europhys. Lett., 30 (1995), pp. 191-196.

[26] A. Evilevitch, L. Lavelle, C. M. Knobler, E. Raspaud, and W. M. Gelbart, Osmotic pressure inhibition of dna ejection from phage, Proceedings of the National Academy of Science, 100 (2003), pp. 9292-9295.

[27] C. P. Fall, E. S. Marland, J. M. Wagner, and J. J. Tyson, eds., Computational cell biology, vol. 20 of Interdisciplinary Applied Mathematics, SpringerVerlag, New York, 2002.

[28] L. J. Fauci And A. L. Fogelson, Truncated Newton Method and the Modeling of Complex Immersed Elastic Structures, Communication on Pure and Applied Mathematics, XLVI (1993), pp. 787-818.

[29] L. Fradkin, Comparison of Lagrangian and Eulerian approaches to turbulent diffusion, Plasma Physics and Controlled Fusion, 33 (1991), pp. 685-701.

[30] García-Archilla, B. and Sanz-Serna, J. M. And Skeel, R. D., Long-timestep methods for oscillatory differential equations, SIAM Journal of Scientific Computing, 20 (1999), pp. 930 - 963.

[31] C. W. Gardiner, Handbook of stochastic methods, Springer Series in Synergetics, 1985

[32] E. Givelberg And J. Bunn, Detailed simulation of the cochlea: Recent progress using large shared memory parallel computers, CACR Technical Report CACR190, (2001).

[33] N. Gov And S. A. SAFran, Red-blood cell membrane fluctuations and shape controlled by ATP-induced cytoskeletal defects, Biophysical Journal BioFAST, (2004).

[34] H. P. Grimm, A. B. Verkhovsky, A. Mogilner, and J. J. Meister, Analysis of actin dynamics at the leading edge of crawling cells: implications for the shape of keratocyte lamellipodia, European Biophysical Journal, 32 (2003), pp. $563-577$.

[35] R. D. Groot and P. B. Warren, Dissipative particle dynamics: Bridging the gap between atomistic and mesoscopic simulation, J. Chem. Phys., 107 (1997), pp. $4423-4435$.

[36] E. Hairer and C. Lubich, Long-Time Energy Conservation of Numerical Methods for Oscillatory Differential Equations, SIAM Journal of Numerical Analysis, 38 (2000), pp. $414-441$.

[37] Hauge, E. H. And Martin-LöF, A., Fluctuating hydrodynamics and Brownian motion, vol. 7, (1973), pp. 259-281.

[38] E. J. Hinch, Application to the Langevin Equation to Fluid Suspensions, Journal of Fluid Mechanics, 72, (1975), pp. 499-511.

[39] P. J. Hoogerbrugge and J. M. V. A. Koelman, Simulating microscopic hydrodynamic phenomena with dissipative particle dynamics, Europhys. Lett., 19 (1992), pp. 155-160.

[40] F. C. Hoppensteadt and C. Peskin, Modeling and Simulation in Medicine and the Life Sciences, Springer (2002). 
[41] J. Howard, Mechanics of Motor Proteins and the Cytoskeleton, Sinauer Associates, Sunderland, CN, 2001.

[42] A. Kassam and L. N. Trefethen, Fourth-Order Time-Stepping for Stiff PDEs, SIAM Journal of Scientific Computing, 26(4) (2005), pp. 1214-1233.

[43] G. Koster and M. Vanduijn and B. Hofs and M. Dogterom, Membrane tube formation from giant vesicles by dynamic association of motor proteins, Proceedings of the National Academies of Science, 100(26) December 2003, pp. $15583-15588$.

[44] P. E. Kloeden and E. Platen, Numerical solution of stochastic differential equations, Springer-Verlag, 1992.

[45] P. R. Kramer And C. S. Peskin, Incorporating thermal fluctuations into the immersed boundary method, Proceedings of the Second MIT Conference on Computational Fluid and Solid Mechanics, K. K. Bathe, ed. Elsevier, 2 (2003), pp. $1755-1758$.

[46] P. R. Kramer and A. J. Majda, Stochastic mode reduction for the immersed boundary method, SIAM J. Appl. Math., 64 (2003/04), pp. 369-400 (electronic).

[47] R. Kubo, M. Toda, and N. Hashitsume, Statistical physics. II, SpringerVerlag, Berlin, second ed., 1991, section 4. Nonequilibrium statistical mechanics.

[48] A. J. C. LADD, Numerical simulations of particulate suspensions via a discretized boltzmann equation. Part 1. Theoretical foundation, J. Fluid Mech., 271 (1994), pp. 285-309.

[49] L. D. Landau And E. M. Lifshitz, Course of theoretical physics. Vol. 9: Statistical physics, Pergamon Press, Oxford, 1980, ch. IX.

[50] L. D. Landau and E. M. Lifshitz, Course of theoretical physics. Vol. 6: Fluid Mechanics, Butterworth-Heinemann, Oxford, 1987, ch. II.

[51] R. Lipowsky, Statistical Mechanics of Biocomplexity, Springer, 1999.

[52] A. J. Majda, I. Timofeyev, and E. Vanden Eijnden, A mathematical framework for stochastic climate models, Comm. Pure Appl. Math., 54 (2001), pp. 891-974.

[53] C. A. Marsh and J. M. Yeomans, Dissipative particle dynamics: The equilibrium for finite time steps, Europhys. Lett., 37 (1997), pp. 511-516.

[54] P. Mazur, Fluctuating Hydrodynamics and Renormalization of Susceptibilities and Transport Coefficient, Fundamental Problems in Statistical Mechanics III, International Summer School on Fundamental Problems in Statistical Mechanics, (1974), pp. 389-414.

[55] X. Meng, A computational model of flow through porous media at the microscale, Ph.D. thesis, Department of Mathematics, Tulane University, New Orleans, Louisiana 70118. July 1998.

[56] L. Miller And C. Peskin, When vortices stick: an aerodynamic transition in tiny insect flight, J Exp Biol., 207 (2004), pp. 3073-3088.

[57] Milstein, G. N. and Repin, Yu. M. and Tretyakov, M. V., Numerical methods for stochastic systems preserving symplectic structure, SIAM Journal of Numerical Analysis, 40 (2002), pp. 1583 - 1604.

[58] P. Nikunen, M. Karttunen, and I. Vattulainen, How would you integrate the equations of motion in dissipative particle dynamics simulations?, Computer Physics Communications, 153 (2003), pp. 407-423.

[59] B. Oksendal, Stochastic Differential Equations: An Introduction with Applications. Springer, 2000

[60] G. Oster And A. MogiLner, Force generation by cellular polymers. 
Supramolecular Polymers, A. Ciferri (ed.), New York:Dekker (in press), 2005.

[61] G. Oster And C. S. Peskin, Dynamics of osmotic fluid flow, in Swelling Mechanics: From Clays to Living Cells and Tissues, T. Karalis, ed., Springer-Verlag, Berlin, 1992, pp. 731-742.

[62] H.C. ÖtTINGER AND Y. RABin, Diffusion Equation versus Coupled Langevin Equations Approach to Hydrodynamics of Dilute Polymer Solutions, Journal of Rheology, 33(5) (1989), pp. 725-743.

[63] I. Pagonabarraga, M. H. J. Hagen, and D. Frenkel, Self-consistent dissipative particle dynamics algorithm, Europhys. Lett., 42 (1998), pp. 377-382.

[64] C. S. Peskin, The immersed boundary method, Acta Numerica, 11 (2002), pp. 139.

[65] C. Peskin And D. MCQueEn, Shared-memory parallel vector implementation of the immersed boundary method for the computation of blood flow in the beating mammalian heart., Journal of Supercomputing, 11 (1997), pp. 213-236.

[66] C. S. Peskin, Cellular Motions and Thermal Fluctuations: The Brownian Ratchet, Biophysical Journal, 65 (1993), pp. 3161-324.

[67] Y. Pomeau And P. RÉsibois, Time Dependent Correlation Functions and Mode-Mode Coupling Theories, Phys. Rep. vol.19C, num. 2 (1975), pp.63-139

[68] W. H. Press and Saul A. Teukolsky and W. T. Vetterling and B. P. Flannery, Numerical Recipes, Cambridge University Press, 2002

[69] A. RAJ, The influence of chromosome flexibility on chromosome transport during anaphase A, Proceedings of National Academy of Sciences, 103(14), April 2006, pp. 5349-5354.

[70] L. E. Reichl, A modern course in statistical physics, John Wiley \& Sons Inc., New York, second ed., 1998

[71] P. Reimann, Introduction to the physics of Brownian motors, Appl. Phys A, 75 (2002), pp. 169-178.

[72] D. N. Robinson And J. A. Spudich, Mechanics and regulation of cytokinesis, Current Opinion in Cell Biology, 16 (2004), pp. 182-188.

[73] J.-N. Roux, Brownian particles at different times scales: a new derivation of the Smoluchowski equation, Phys. A, 188 (1992), pp. 526-552.

[74] J. M. Sanz-Serna And M. P. Calvo, Numerical Hamiltonian problems, Chapman \& Hall, 1994

[75] T. Schlick, Molecular Modeling and Simulation: An Interdisciplinary Guide, vol. 21 of Interdisciplinary Applied Mathematics, Springer-Verlag, Berlin, 2002.

[76] N. Sharma And N. A. Patankar, Direct numerical simulation of the brownian motion of particles by using fluctuating hydrodynamic equations, J. Comp. Phys, 201 (2004), pp. 466-486.

[77] A. Sierou and J. F. Brady, Accelerated Stokesian Dynamics simulations, J. Fluid Mech., 448 (2001), pp. 115-146.

[78] K. Berg-Sorensen and H. Flyvbjerg, The colour of thermal noise in classical Brownian motion: a feasibility study of direct experimental observation, New Journal of Physics, 7 (2005)

[79] M. Talagrand, Sharper bounds for gaussian and empirical processes, Annals of Probability, 22 (1994), pp. 28-76.

[80] D. Talay, Simulation of stochastic processes and applications in Foundations of computational mathematics, Cambridge Univ. Press, 284, (2001) pp. 345-359.

[81] J. A. TheRIOt, The polymerization motor, Traffic, 1 (2000), pp. 19-28.

[82] J. WeInstock, Lagrangian Eulerian relation and the independence approxima- 
tion, Physics of Fluids, 19 (1976), pp. 1702-1711.

[83] C. Wolgemuth, A. Mogilner and G. Oster, The hydration dynamics of polyelectrolyte gels with applications to cell motility and drug delivery, European Biophysics Journal, 33 (2004), pp. 146-158.

[84] T. Wittman, A. Hyman, And A. Desai, The spindle: a dynamic assembly of microtubules and motors, Nature Cell Biology, 3 (2001), pp. E28 - E34. 
Tables. 
TABLE 4.1

Parameters of the Method

\begin{tabular}{|l|l|}
\hline Parameter & Description \\
\hline$k_{B}$ & Boltzmann's constant \\
$T$ & Temperature \\
$L$ & Period Length of Fluid Domain \\
$\mu$ & Fluid Dynamic Viscosity \\
$\rho$ & Fluid Density \\
$N$ & Number of Grid Points in each Dimension \\
$\Delta t$ & Time Step \\
$\Delta x$ & Space Between Grid Points \\
$a$ & Effective Elementary Particle Size (approximate radius) \\
\hline
\end{tabular}

TABLE 4.2

Values used in Numerical Simulations

\begin{tabular}{|l|l|}
\hline Parameter & Description \\
\hline$T$ & $300 \mathrm{~K}$ \\
$L$ & $1000 \mathrm{~nm}$ \\
$\mu$ & $6.02 \times 10^{5} \mathrm{amu} /(\mathrm{nm} \cdot \mathrm{ns})$ \\
$\rho$ & $602 \mathrm{amu} / \mathrm{nm}^{3}$ \\
$N$ & 32 \\
\hline
\end{tabular}


TABle 4.3

Notation Conventions

\begin{tabular}{|l|l|}
\hline Parameter & Description \\
\hline$\delta_{a}$ & Representation function of an immersed elementary particle of size $a$ \\
$\delta_{a, \mathbf{k}}$ & The $\mathbf{k}^{t h}$ Fourier coefficient of the particle representation function \\
$\alpha_{\mathbf{k}}$ & Damping of the $\mathbf{k}^{t h}$ Fourier mode \\
$D_{\mathbf{k}}$ & Strength of the thermal forcing of the $\mathbf{k}^{t h}$ Fourier mode \\
$\mathbf{u}_{m}$ & Fluid velocity at the $\mathbf{m}^{t h}$ grid point \\
$\hat{\mathbf{u}}_{k}$ & The $\mathbf{k}^{t h}$ Fourier mode of the fluid velocity field \\
$\mathbf{U}$ & Smoothed fluid velocity field for immersed elementary particles \\
$\mathbf{x}_{m}$ & Position vector of the $\mathbf{~}^{t h}$ Eulerian grid point \\
$\mathbf{X}^{[j]}$ & Position vector of the $j^{t h}$ immersed elementary particle \\
$\mathbf{f}_{\text {prt }}$ & Force density arising from the immersed structures \\
$\mathbf{f}_{\mathrm{thm}}$ & Force density arising from the thermal forcing \\
$\hat{\mathbf{f}}_{k}$ & The $\mathbf{k}^{t h}$ Fourier mode of the structural force density field \\
\hline
\end{tabular}

